\title{
IMPLICATIONAL RELEVANCE LOGIC IS 2-EXPTIME-COMPLETE
}

\author{
SYLVAIN SCHMITZ
}

\begin{abstract}
We show that provability in the implicational fragment of relevance logic is complete for doubly exponential time, using reductions to and from coverability in branching vector addition systems.

KEY WordS. Relevance logic, branching VASS, focusing proofs, complexity
\end{abstract}

\section{INTRODUCTION}

Relevance logic $\mathbf{R}$ [1, 12] provides a formalisation of 'relevant' implication: in such a system, the formula $A \rightarrow B$ indicates that the truth of $A$ is actually useful in establishing $B$; an example of an irrelevant implication valid in classical logic would be $B \rightarrow(A \rightarrow B)$.

The pure implicational fragment $\mathbf{R}_{\rightarrow}$ of $\mathbf{R}$ was developed independently by Moh [19] in 1950 and Church [6] in 1951, and is as such the oldest of the relevance logics. Kripke already presented in 1959 a decision algorithm for provability in $\mathbf{R} \rightarrow$ [14], which was later extended to larger and larger subsets of $\mathbf{R}$, like the conjunctive-implicational fragment $\mathbf{R}_{\rightarrow, \wedge}$. Several negative results by Urquhart would however foil any hope for elementary algorithms: first in 1984 when he showed the undecidability of the full logic $\mathbf{R}$ [26] ; later in 1999 with a proof that $\mathbf{R}_{\rightarrow, \wedge}$ suffers from a non primitiverecursive complexity: it is ACKERMANN-complete [28]. This left a gigantic gap for the implicational fragment $\mathbf{R}_{\rightarrow}$, between an earlier ExPSPACE lower bound [27] and the ACKERMANN upper bound shared by the variants of Kripke's procedure.

In this paper, we close this gap and show that provability in $\mathbf{R}_{\rightarrow}$ is 2-ExPTIME-complete. Our proof relies crucially on a recent result by Demri et al. [10], who show the 2-EXPTIME-completeness of the coverability problem in branching vector addition systems with states (BVASS). These systems form a natural generalisation of vector addition systems, and have been defined independently in a variety of contexts (see the survey [24] and Section 3 below), notably that of provability in multiplicative exponential linear logic (MELL, see [9]). More precisely:

- In Section 4, we show that so-called expansive BVASSs can simulate proofs in $\mathbf{R}_{\rightarrow}$ in a natural manner by exploiting the subformula property of its usual sequent calculus $L \mathbf{R}_{\rightarrow}$. We then show how to reduce reachability in expansive BVASS to coverability, thereby providing a decision procedure in doubly exponential time. 
- The matching hardness proof in Section 5 relies on the one hand on comprehensive instances of the BVASS coverability problem, and on the other hand on a new focusing sequent calculus $F \mathbf{R}_{\rightarrow}$ for $\mathbf{R}_{\rightarrow}$.

- The reduction from $\mathbf{R}_{\rightarrow}$ provability to expansive BVASS reachability is actually a special case of a more general reduction proved in [15] for the multiplicative exponential fragment of intuitionistic contractive linear logic (IMELLC), i.e. IMELL with structural contraction. Our reduction in Section 4.3 from expansive reachability to coverability thus entails that IMELLC provability is 2-ExPTIMEcomplete, as explained in Section 6 .

Let us first recall the formal definition of $\mathbf{R}_{\rightarrow}$ before turning to that of BVASSs in Section 3.

\section{The Implicational Fragment $\mathbf{R}_{\rightarrow}$}

The reader will find in [12, Section 4] a nice overview of the decision problem for $\mathbf{R}$, covering in particular Kripke's solution for $\mathbf{R}_{\rightarrow}$ [14] and Urquhart]'s lower bound argument for $\mathbf{R}_{\rightarrow, \wedge}[28]$.

2.1. A Sequent Calculus. We recall here the formal definition of $\mathbf{R}_{\rightarrow}$ as a sequent calculus $L \mathbf{R}_{\rightarrow}$ in Gentzen's style. Let $\mathcal{A}$ be a countable set of atomic propositions; we define the set of formulæ as following the abstract syntax

$$
A::=a \mid A \rightarrow A \quad \text { (implicational formulæ) }
$$

where $a$ ranges over $\mathcal{A}$. We consider $\rightarrow$ to be right-associative, e.g. $A \rightarrow$ $B \rightarrow C$ denotes $A \rightarrow(B \rightarrow C)$. In the following rules, we use $A, B, C, \ldots$ to denote implicational formulæ and $\Gamma, \Delta, \ldots$ to denote multisets of such formulæ; commas in e.g. ' $\Gamma, A$ ' and ' $\Gamma, \Delta$ ' denote multiset unions of $\Gamma$ with the singleton $A$ and with $\Delta$ respectively; finally, a sequent is a pair ' $\Gamma \vdash$ $A$ ' stating that the succedent $A$ is valid assuming the antecedent $\Gamma$ to be relevant:

$$
\begin{array}{cc}
\frac{}{A \vdash A}(\mathrm{ld}) & \frac{\Gamma, A, A \vdash B}{\Gamma, A \vdash B}(\mathrm{C}) \\
\frac{\Gamma \vdash A \quad \Delta, B \vdash C}{\Gamma, \Delta, A \rightarrow B \vdash C}(\rightarrow \mathrm{L}) & \frac{\Gamma, A \vdash B}{\Gamma \vdash A \rightarrow B}\left(\rightarrow_{\mathrm{R}}\right)
\end{array}
$$

As we work with multisets, this sequent calculus includes implicitly the structural 'exchange' rule. It does however not feature the classical 'weakening' rule - which would defeat the very point of relevance - nor the 'cut' rule - which is admissible. A visible consequence of this definition is that the calculus enjoys the subformula property: all the formulæ in rule premises are subformulæ of the formulæ appearing in the corresponding consequences.

2.2. Decidability and Complexity. With hindsight, the decision procedure of Kripke [14 for provability in the implicational fragment of relevance logic can be seen as a precursor for many later algorithms that rely on the existence of a well quasi ordering (wqo) for their termination [22]. This decision procedure can be understood as an application of Dickson's Lemma to prove the finiteness of 'irredundant' proof trees for the target sequent $\vdash A$. 
Furthermore, combinatorial analyses of Dickson's Lemma as e.g. in [13] provide explicit upper bounds on the size of those irredundant proofs, in the form of the Ackermann function in the size of $A$, yielding an ACKERmann upper bound for $\mathbf{R}_{\rightarrow}$ provability, as shown by Urquhart [28].

Regarding lower bounds, Urquhart in [27, Section 9] explains how to derive EXPSPACE-hardness for $\mathbf{R}_{\rightarrow}$, using model-theoretic techniques to reduce from the word problem for finitely presented commutative semigroups [18].

2.3. Strict $\boldsymbol{\lambda}$-Calculus. The implicational fragment $\mathbf{R}_{\rightarrow}$ is in bijection with the typing rules of the simply typed $\lambda I$-calculus, where abstracted terms $\lambda$ x.t are well-formed only if $x$ appears free in $t$; see [7, Section 9F]. This means that $\mathbf{R}_{\rightarrow}$ provability can be restated as the type inhabitation problem for the simply typed $\lambda I$-calculus. Our complexity results should then be contrasted with the PSPACE-completeness of the same problem for the simply typed $\lambda$-calculus [25].

\section{BRANCHING VASS}

Branching vector addition systems with states (hereafter BVASS) have been independently defined in several contexts:

- in computational linguistics 24, as a means of modelling grammatical dependencies between tree nodes in unordered vector grammars with dominance edges [21] - they have since been related with $a b$ stract categorial grammars [8] and minimalist grammars [23] -

- in computational logic, as a way to attack the decision problem for MELL through counter machines [9],

- in cryptographic protocol verification, as a means to reason in rewriting systems modulo associativity and commutativity of some symbols [29].

They have furthermore been linked to the satisfiability problem of twovariables FO over data trees [4, 11] and the modelling of parallel programming libraries [5].

3.1. Formal Definitions. Given $d$ in $\mathbb{N}$, we write ' $\overline{0}$ ' for the null vector in $\mathbb{N}^{d}$, and for $0<i \leq d$, ' $\overline{\mathrm{e}}_{i}$ ' for the unit vector in $\mathbb{N}^{d}$ with 1 on coordinate $i$ and 0 everywhere else. Let $U_{d} \stackrel{\text { def }}{=}\left\{\overline{\mathrm{e}}_{i},-\overline{\mathrm{e}}_{i} \mid 0<i \leq d\right\}$. Syntactically, an ordinary $B V A S S$ is a tuple $\mathcal{B}=\left\langle Q, d, T_{u}, T_{s}\right\rangle$ where $Q$ is a finite set of states, $d$ is a dimension in $\mathbb{N}$, and $T_{u} \subseteq Q \times U_{d} \times Q$ and $T_{s} \subseteq Q^{3}$ are respectively finite sets of unary and split rules. We denote unary rules $\left(q, \bar{u}, q_{1}\right)$ in $T_{u}$ with $\bar{u}$ in $U_{d}$ by ' $q \stackrel{\bar{u}}{\rightarrow} q_{1}$ ' and split rules $\left(q, q_{1}, q_{2}\right)$ in $T_{s}$ by ' $q \rightarrow q_{1}+q_{2}$ '.

We define the semantics of an ordinary BVASS through a deduction system over configurations $(q, \overline{\mathrm{v}})$ in $Q \times \mathbb{N}^{d}$ :

$$
\frac{q, \overline{\mathrm{v}}}{q_{1}, \overline{\mathrm{v}}+\overline{\mathrm{e}}_{i}} \text { (incr) } \quad \frac{q, \overline{\mathrm{v}}+\overline{\mathrm{e}}_{i}}{q_{1}, \overline{\mathrm{v}}} \text { (decr) } \quad \frac{q, \overline{\mathrm{v}}_{1}+\overline{\mathrm{v}}_{2}}{q_{1}, \overline{\mathrm{v}}_{1} q_{2}, \overline{\mathrm{v}}_{2}} \text { (split) }
$$

respectively for unary rules $q \stackrel{\overline{\mathrm{e}}_{i}}{\longrightarrow} q_{1}$ and $q \stackrel{-\overline{\mathrm{e}}_{i}}{\longrightarrow} q_{1}$ in $T_{u}$ and a split rule $q \rightarrow q_{1}+q_{2}$ in $T_{s}$; in split ' + ' denotes component-wise addition in $\mathbb{N}^{d}$. Such a deduction system can be employed either top-down or bottom-up depending on the decision problem at hand (as with tree automata); the 
top-down direction will correspond in a natural way to goal-directed proof search in the sequent calculus of Section 2 .

3.1.1. Ordinary BVASSs are a slight restriction over BVASSs, which would in general allow any vector in $\mathbb{Z}^{d}$ in unary rules. Because they often lead to more readable proofs, we only employ ordinary BVASSs in this paper. This is at no loss of generality, since one can build an ordinary BVASS 'equivalent' to a given BVASS in logarithmic space, where equivalence should be understood relative to the reachability and coverability problems; see [24] for details.

3.1.2. Reachability. Branching VASSs are associated with a natural decision problem: reachability asks, given a BVASS $\mathcal{B}$, a root state $q_{r}$, and a leaf state $q_{\ell}$, whether there exists a deduction tree with root label $\left(q_{r}, \overline{0}\right)$ and every leaf labelled $\left(q_{\ell}, \overline{0}\right)$; such a deduction tree is called a reachability witness. De Groote et al. [9] have shown that this problem is recursively equivalent to MELL provability, and it is currently unknown whether it is decidableboth problems are however known to be of non-elementary computational complexity [15].

Let us introduce some additional notation that will be handy in proofs. We write ' $\mathcal{B}, T, q_{\ell} \triangleright q, \overline{\mathrm{v}}$ ' if there exists a deduction tree of $\mathcal{B}$ with root label $(q, \overline{\mathrm{v}})$ and leaves labelled by $\left(q_{\ell}, \overline{0}\right)$, which uses each rule in $T \subseteq T_{u} \uplus T_{s}$ at least once. Such root judgements can be derived through the deduction system

$$
\begin{aligned}
& \overline{\mathcal{B}, \emptyset, q_{\ell} \triangleright q_{\ell}, \overline{0}} \quad \frac{\mathcal{B}, T, q_{\ell} \triangleright q_{1}, \overline{\mathrm{v}}+\overline{\mathrm{e}}_{i}}{\mathcal{B}, T \cup\left\{q \stackrel{\mathrm{e}_{i}}{\rightarrow} q_{1}\right\}, q_{\ell} \triangleright q, \overline{\mathrm{v}}} \\
& \frac{\mathcal{B}, T, q_{\ell} \triangleright q_{1}, \overline{\mathrm{v}}}{\mathcal{B}, T \cup\left\{q \stackrel{-\overline{\mathrm{e}}_{i}}{\longrightarrow} q_{1}\right\}, q_{\ell} \triangleright q, \overline{\mathrm{v}}+\overline{\mathrm{e}}_{i}} \quad \frac{\mathcal{B}, T_{1}, q_{\ell} \triangleright q_{1}, \overline{\mathrm{v}}_{1} \quad \mathcal{B}, T_{2}, q_{\ell} \triangleright q_{2}, \overline{\mathrm{v}}_{2}}{\mathcal{B}, T_{1} \cup T_{2} \cup\left\{q \rightarrow q_{1}+q_{2}\right\}, q_{\ell} \triangleright q, \overline{\mathrm{v}}_{1}+\overline{\mathrm{v}}_{2}}
\end{aligned}
$$

We write more simply ' $\mathcal{B}, q_{\ell} \triangleright q, \bar{v}$ ' if there exists $T \subseteq T_{u} \uplus T_{s}$ such that $\mathcal{B}, T, q_{\ell} \triangleright q, \bar{v}$. With these notations, the reachability problem asks whether $\mathcal{B}, q_{\ell} \triangleright q_{r}, \overline{0}$.

3.2. Root Coverability. Our interest in this paper lies in a relaxation of the reachability problem, where we ask instead to cover the root: given as before $\left\langle\mathcal{B}, q_{r}, q_{\ell}\right\rangle$, we ask whether there exists a coverability witness, i.e. a deduction tree with root $\left(q_{r}, \overline{\mathrm{v}}\right)$ for some $\overline{\mathrm{v}}$ in $\mathbb{N}^{d}$ and leaves $\left(q_{\ell}, \overline{0}\right)$; in other words whether $\mathcal{B}, q_{\ell} \triangleright q_{r}, \overline{\mathrm{v}}$ for some $\overline{\mathrm{v}}$ in $\mathbb{N}^{d}$.

This problem was shown decidable by Verma and Goubault-Larrecq [29], and was later proven 2-ExPTIME-complete by Demri et al. [10] in a slight variant called branching vector addition systems (BVAS):

Fact 3.1 (10, Theorem 8 and Theorem 21). BVAS coverability is 2-ExPTIMEcomplete.

Branching VAS are not equipped with a state space $Q$. Their coverability problem is stated slightly differently, but is easy to reduce in both directions to BVASS coverability. This would not be worth mentioning here if it were not for the following instrumental corollary of their proof, which exploits an encoding of $d$-dimensional BVASSs into $(d+6)$-dimensional BVASs: 
Corollary 3.2. Coverability in a $B V A S S \mathcal{B}=\left\langle Q, d, T_{u}, T_{s}\right\rangle$ can be solved

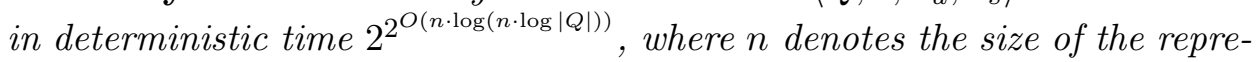
sentation of $\left\langle d, T_{u}\right\rangle$.

Corollary 3.2 entails that coverability remains in 2-ExPTIME for BVASSs with double exponential state space. This is an easy result, which we show in Appendix B.

\section{UPPER BOUND}

In order to show a 2-EXPTime upper bound for $\mathbf{R}_{\rightarrow}$ provability, we introduce as an intermediate decision problem the expansive reachability problem for BVASS (Section 4.1). Then, the first step of our proof in Section 4.2 takes us from the sequent calculus $L \mathbf{R} \rightarrow$ to reachability in expansive BVASS. This is a simple construction that relies on the subformula property of $L \mathbf{R}_{\rightarrow}$, and is actually a particular case of a more general reduction shown in [15, Proposition 9]. The new technical result here is the second step: a reduction from expansive BVASS reachability to BVASS coverability, which is shown in Section 4.3. This new reduction also entails new upper bounds for provability in extensions of $L \mathbf{R}_{\rightarrow}$ studied in [15]; see Section 6.

4.1. Expansive Reachability. An expansive BVASS is a BVASS with an additional deduction rule:

$$
\frac{q, \overline{\mathrm{v}}+\overline{\mathrm{e}}_{i}}{q, \overline{\mathrm{v}}+2 \overline{\mathrm{e}}_{i}}(\text { expansion})
$$

Note that expansions could be simulated by unary rules $q \stackrel{-\overline{\mathrm{e}}_{i}}{\longrightarrow} q_{i} \stackrel{\overline{\mathrm{e}}_{i}}{\rightarrow} q_{i}^{\prime} \stackrel{\overline{\mathrm{e}}_{i}}{\rightarrow} q$ for all $q$ in $Q$ and $0<i \leq d$; we prefer to see them as new deduction rules.

This yields a new rule for root judgements, which we denote using ' $\nabla_{e}$ ' to emphasise that we allow expansion rules:

$$
\frac{\mathcal{B}, T, q_{\ell} \triangleright_{e} q, \overline{\mathrm{v}}+2 \overline{\mathrm{e}}_{i}}{\mathcal{B}, T, q_{\ell} \triangleright_{e} q, \overline{\mathrm{v}}+\overline{\mathrm{e}}_{i}}
$$

The expansive reachability problem then asks, given an expansive BVASS $\mathcal{B}$ and two states $q_{r}$ and $q_{\ell}$, whether $\mathcal{B}, q_{\ell} \triangleright_{e} q_{r}, \overline{0}$.

4.2. From $\boldsymbol{L} \mathbf{R}_{\rightarrow}$ to Expansive Reachability. We prove here the following reduction:

Proposition 4.1. There is a logarithmic space reduction from provability in $\mathbf{R}_{\rightarrow}$ to expansive reachability in ordinary BVASSs.

Let us consider an instance $\langle F\rangle$ of the provability problem for $\mathbf{R}_{\rightarrow}$ for an implicational formula $F$. The instance is positive if and only if we can find a proof for $\vdash F$ in $L \mathbf{R}_{\rightarrow}$. Thanks to the subformula property, we know that in such a proof, all the sequents $\Gamma \vdash A$ must use subfomulæ of $F$. That is, if we denote by $S$ the set of subformulæ of $F$, then $\Gamma$ is in $\mathbb{N}^{S}$ and $A$ in $S$.

We construct from $F$ an expansive BVASS $\mathcal{B}_{F}$ that implements proof search in $L \mathbf{R}_{\rightarrow}$ restricted to subformulæ of $F$. We define for this $\mathcal{B}_{F} \stackrel{\text { def }}{=}$ $\left\langle Q_{F},|S|, T_{u}, T_{s}\right\rangle$ where the state space $Q_{F}$ includes $S$ and a distinguished leaf state $q_{\ell}$. It also includes some intermediate states as introduced in the translations of the rules of $L \mathbf{R}_{\rightarrow}$ into rules in $T_{u} \cup T_{s}$ depicted in Figure 1 . 


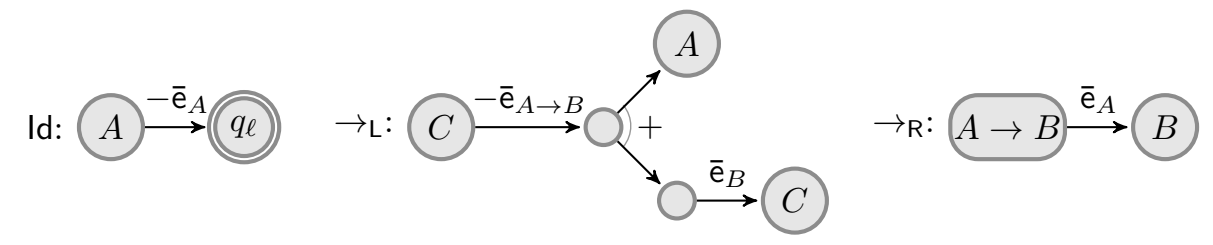

FiguRE 1. The rules and intermediate states of $\mathcal{B}_{F}$.

Note that (C) has no associated rule; it relies instead on expansions in $\mathcal{B}_{F}$. The full state space $Q_{F}$ of $\mathcal{B}_{F}$, including intermediate states, is thus of size $O\left(|F|^{2}\right)$.

Let us write $\bar{v}_{\Gamma}$ for the vector in $\mathbb{N}^{|S|}$ associated with a multiset $\Gamma$ in $\mathbb{N}^{S}$. The proof of Proposition 4.1 is a consequence of the following claim instantiated with $A=F$ and $\Gamma=\emptyset$ :

Claim 4.2. For all $\Gamma$ in $\mathbb{N}^{S}$ and $A$ in $S, \Gamma \vdash A$ if and only if $\mathcal{B}_{F}, q_{\ell} \triangleright_{e} A, \bar{v}_{\Gamma}$.

Proof. We proceed by induction of the structure of proofs in $L \mathbf{R}_{\rightarrow}$ and of expansive root judgements in $\mathcal{B}_{F}$.

identity; for the base case (Id), $A \vdash A$ iff $\mathcal{B}_{F}, q_{\ell} \triangleright_{e} A, \overline{\mathrm{e}}_{A}$.

contraction; If $\Gamma, A \vdash B$ as the result of (C) from $\Gamma, A, A \vdash B$ then by induction hypothesis $\mathcal{B}_{F}, q_{\ell} \triangleright_{e} B, \bar{v}_{\Gamma, A, A}$, from which an expansion yields $\mathcal{B}_{F}, q_{\ell} \triangleright_{e} B, \bar{v}_{\Gamma, A}$. Conversely, if $\mathcal{B}_{F}, q_{\ell} \triangleright_{e} B, \bar{v}_{\Gamma, A}$ as the result of an expansion from $\mathcal{B}_{F}, q_{\ell} \triangleright_{e} B, \bar{v}_{\Gamma, A, A}$, then by induction hypothesis $\Gamma, A, A \vdash B$ and a contraction yields $\Gamma, A \vdash B$.

left implication: If $\Gamma, \Delta, A \rightarrow B \vdash C$ as the result of $\rightarrow_{\mathrm{L}}$ applied to $\Gamma \vdash A$ and $\Delta, B \vdash C$, then by induction hypothesis $\mathcal{B}_{F}, q_{\ell} \triangleright_{e} A, \bar{v}_{\Gamma}$ and $\mathcal{B}_{F}, q_{\ell} \triangleright_{e} C, \bar{v}_{\Delta, B}$. Then the group of rules in $\mathcal{B}_{F}$ yields $\mathcal{B}_{F}, q_{\ell} \triangleright_{e}$ $C_{B}, \bar{v}_{\Delta}$ and $\mathcal{B}_{F}, q_{\ell} \triangleright_{e} C_{A \rightarrow B}, \bar{v}_{\Gamma, \Delta}$ in the intermediate states, and finally $\mathcal{B}_{F}, q_{\ell} \triangleright_{e} C, \bar{v}_{\Gamma, \Delta, A \rightarrow B}$ as desired. The converse direction is similar.

right implication: If $\Gamma \vdash A \rightarrow B$ as the result of $\left(\rightarrow_{\mathrm{R}}\right.$ applies to $\Gamma, A \vdash B$, then by induction hypothesis $\mathcal{B}_{F}, q_{\ell} \triangleright_{e} B, \bar{v}_{\Gamma, A}$, from which the corresponding rule of $\mathcal{B}_{F}$ yields $\mathcal{B}_{F}, q_{\ell} \triangleright_{e} A \rightarrow B, \bar{v}_{\Gamma}$ as desired. The converse direction is similar.

4.3. From Expansive Reachability to Coverability. The second step of our proof that $\mathbf{R}_{\rightarrow}$ provability is in 2-ExPTIME is then to reduce expansive reachability to coverability in BVASS. Our reduction incurs an exponential blow-up in the number of states, but thanks to Corollary 3.2, this still results in a 2-ExPTIME algorithm:

Proposition 4.3. There is a polynomial space reduction from BVASS expansive reachability to BVASS coverability.

4.3.1. Topmost Increments. Consider an instance $\left\langle\mathcal{B}, q_{r}, q_{\ell}\right\rangle$ of the expansive reachability problem with $\mathcal{B}=\left\langle Q, d, T_{u}, T_{s}\right\rangle$. Because the root vector of an expansive reachability witness must be $\overline{0}$, we can identify along each branch of the witness and for each coordinate $0<i \leq d$ the topmost (i.e. closest to the root) application of an (incr) rule - possibly no such increment ever occurs on some branches. 
Assume without loss of generality that $q_{\ell}$ has no outgoing transition in $\mathcal{B}$. We construct a new BVASS $\mathcal{B}^{\dagger}=\left\langle Q^{\dagger}, d, T_{u}^{\dagger}, T_{s}\right\rangle$ with additional states $q_{\ell}^{i}$ and unary rules $q_{\ell} \stackrel{\overline{\mathrm{e}}_{i}}{\rightarrow} q_{\ell}^{i} \stackrel{-\overline{\mathrm{e}}_{i}}{\longrightarrow} q_{\ell}$ for every $0<i \leq d$. Then $\mathcal{B}, q_{\ell} \triangleright_{e} q_{r}, \overline{0}$ if and only if $\mathcal{B}^{\dagger}, q_{\ell} \triangleright_{e} q_{r}, \overline{0}$ (observe in particular that no expansion in $q_{\ell}^{i}$ can occur in an expansive reachability witness). Additionally, the new rules allow us to assume that there is a topmost increment for each branch and every coordinate of an expansive reachability witness of $\mathcal{B}^{\dagger}$.

Let $[d] \stackrel{\text { def }}{=}\{1, \ldots, d\}$. The root judgement relation can be refined as ' $\triangleright_{e}^{s}$, with a set $s \subseteq[d]$ of coordinates. The intended semantics for $i \in s$ is that there is at least one increment on coordinate $i$ earlier on the path from the root in the expansive reachability witness. Formally, at the leaves

$$
\overline{\mathcal{B}^{\dagger}, \emptyset, q_{\ell} \triangleright_{e}^{[d]} q_{\ell}, \overline{0}}
$$

since by assumption every coordinate must see an increase. Then, an increment is either topmost or not:

$$
\frac{\mathcal{B}^{\dagger}, T, q_{\ell} \triangleright_{e}^{s \uplus 4\{i\}} q_{1}, \overline{\mathrm{w}}+\overline{\mathrm{e}}_{i}}{\mathcal{B}^{\dagger}, T \cup\left\{q \stackrel{\overline{\mathrm{e}}_{i}}{\rightarrow} q_{1}\right\}, q_{\ell} \triangleright_{e}^{s} q, \overline{\mathrm{w}}} \quad \frac{\mathcal{B}^{\dagger}, T, q_{\ell} \triangleright_{e}^{s \cup\{i\}} q_{1}, \overline{\mathrm{v}}+\overline{\mathrm{e}}_{i}}{\mathcal{B}^{\dagger}, T \cup\left\{q \stackrel{\overline{\mathrm{e}}_{i}}{\rightarrow} q_{1}\right\}, q_{\ell} \triangleright_{e}^{s \cup\{i\}} q, \overline{\mathrm{v}}}
$$

where $\overline{\mathrm{w}}(i)=0$ and ' $\uplus$ ' denotes disjoint union. Decrements and expansions are necessarily dominated by the topmost increment:

$$
\frac{\mathcal{B}^{\dagger}, T, q_{\ell} \triangleright_{e}^{s \cup\{i\}} q_{1}, \overline{\mathrm{v}}}{\mathcal{B}^{\dagger}, T \cup\left\{q \stackrel{-\overline{\mathrm{e}}_{i}}{\longrightarrow} q_{1}\right\}, q_{\ell} \triangleright_{e}^{s \cup\{i\}} q, \overline{\mathrm{v}}+\overline{\mathrm{e}}_{i}} \quad \frac{\mathcal{B}^{\dagger}, T, q_{\ell} \triangleright_{e}^{s \cup\{i\}} q, \overline{\mathrm{v}}+2 \overline{\mathrm{e}}_{i}}{\mathcal{B}^{\dagger}, T, q_{\ell} \triangleright_{e}^{s \cup\{i\}} q, \overline{\mathrm{v}}+\overline{\mathrm{e}}_{i}}
$$

Finally, the same topmost increments have been seen on both branches of a split:

$$
\frac{\mathcal{B}^{\dagger}, T_{1}, q_{\ell} \triangleright_{e}^{s} q_{1}, \overline{\mathrm{v}}_{1} \quad \mathcal{B}^{\dagger}, T_{2}, q_{\ell} \triangleright_{e}^{s} q_{2}, \overline{\mathrm{v}}_{2}}{\mathcal{B}^{\dagger}, T_{1} \cup T_{2} \cup\left\{q \rightarrow q_{1}+q_{2}\right\}, q_{\ell} \triangleright_{e}^{s} q, \overline{\mathrm{v}}_{1}+\overline{\mathrm{v}}_{2}}
$$

The refined root judgements verify

$$
\mathcal{B}, q_{\ell} \triangleright_{e} q_{r}, \overline{0} \text { implies } \mathcal{B}^{\dagger}, q_{\ell} \triangleright_{e}^{\emptyset} q_{r}, \overline{0},
$$

the converse implication being immediate by removing the ' $s$ ' annotations.

4.3.2. Reduction to Coverability. We construct yet another BVASS $\mathcal{B}^{\ddagger}=$ $\left\langle Q^{\dagger} \times 2^{[d]}, d, T_{u}^{\ddagger}, T_{s}^{\ddagger}\right\rangle$ and build a coverability instance $\left\langle\mathcal{B}^{\ddagger},\left(q_{r}, \emptyset\right),\left(q_{\ell},[d]\right)\right\rangle$. The idea is to maintain a set $s \subseteq[d]$ as in the refined judgements $\triangleright_{e}^{s}$; however since we cannot test for zero we will rely instead on nondeterminism. Let

$$
\begin{array}{rlrl}
T_{u}^{\ddagger} \stackrel{\text { def }}{=}\left\{(q, s) \stackrel{\overline{\mathrm{e}}_{i}}{\rightarrow}\left(q_{1}, s \cup\{i\}\right) \mid q \stackrel{\overline{\mathrm{e}}_{i}}{\rightarrow} q_{1} \in T_{u}^{\dagger}, s \subseteq[d]\right\} & & \left(\text { incr }^{\ddagger}\right) \\
& \cup\left\{(q, s \cup\{i\}) \stackrel{-\overline{\mathrm{e}}_{i}}{\longrightarrow}\left(q_{1}, s \cup\{i\}\right) \mid q \stackrel{-\overline{\mathrm{e}}_{i}}{\longrightarrow} q_{1} \in T_{u}^{\dagger}, s \subseteq[d]\right\}, & & \left(\text { decr }^{\ddagger}\right) \\
T_{s}^{\ddagger} \stackrel{\text { def }}{=}\left\{(q, s) \rightarrow\left(q_{1}, s\right)+\left(q_{2}, s\right) \mid q \rightarrow q_{1}+q_{2} \in T_{s}^{\dagger}, s \subseteq[d]\right\} . & & \left(\text { split }^{\ddagger}\right)
\end{array}
$$

Claim 4.4. If $\mathcal{B}^{\dagger}, q_{\ell} \triangleright_{e}^{s}, q, \overline{\mathrm{v}}$, then there exists $\overline{\mathrm{v}}^{\prime} \geq \overline{\mathrm{v}}$ such that $\mathcal{B}^{\ddagger},\left(q_{\ell},[d]\right) \triangleright$ $(q, s), \overline{\mathrm{v}}^{\prime}$.

Proof. We prove the claim by induction over the root judgement in $\mathcal{B}^{\dagger}$. For the base case with $q=q_{\ell}$ and $\bar{v}=\overline{0}$, we choose $\bar{v}^{\prime}=\overline{0}$. 
For the induction step, if the judgement results from the application of an increment rule on coordinate $i$, then $\mathcal{B}^{\dagger}, q_{\ell} \triangleright_{e}^{s \cup\{i\}} q_{1}, \overline{\mathrm{v}}+\overline{\mathrm{e}}_{i}$, and by induction hypothesis there exists $\bar{v}^{\prime} \geq v$ such that $\mathcal{B}^{\ddagger},\left(q_{\ell},[d]\right) \triangleright\left(q_{1}, s \cup\{i\}\right), \overline{\mathrm{v}}^{\prime}+\overline{\mathrm{e}}_{i}$. Two cases arise depending on whether $i \notin s$ or $i \in s$, i.e. whether this is the topmost increment on coordinate $i$ or not. In both cases, incr yields $\mathcal{B},\left(q_{\ell},[d]\right) \triangleright(q, s), \bar{v}^{\prime}$ as desired.

If the judgement results from the application of a decrement to $\mathcal{B}^{\dagger}, q_{\ell} \triangleright_{e}^{s \cup\{i\}}$ $q_{1}, \bar{v}$, then by induction hypothesis there exists $\bar{v}^{\prime} \geq \bar{v}$ such that $\mathcal{B}^{\ddagger},\left(q_{\ell},[d]\right) \triangleright$ $\left(q_{1}, s \cup\{i\}\right), \bar{v}^{\prime}$, and decr $^{\ddagger}$ shows $\mathcal{B}^{\ddagger},\left(q_{\ell},[d]\right) \triangleright(q, s \cup\{i\}), \overline{\mathrm{v}}^{\prime}+\overline{\mathrm{e}}_{i}$ where $\overline{\mathrm{v}}^{\prime}+\overline{\mathrm{e}}_{i} \geq \overline{\mathrm{v}}+\overline{\mathrm{e}}_{i}$ as desired.

If the judgement results from an expansion applied to $\mathcal{B}^{\dagger}, q_{\ell} \triangleright_{e}^{s \cup\{i\}} q, \bar{v}+$ $2 \overline{\mathrm{e}}_{i}$, then by induction hypothesis there exists $\overline{\mathrm{v}}^{\prime} \geq \overline{\mathrm{v}}+2 \overline{\mathrm{e}}_{i} \geq \overline{\mathrm{v}}+\overline{\mathrm{e}}_{i}$ and $\mathcal{B}^{\ddagger},\left(q_{\ell},[d]\right) \triangleright(q, s \cup\{i\}), \overline{\mathrm{v}}^{\prime}$ as desired.

Finally, if the judgement results from a split with premises $\mathcal{B}^{\dagger}, q_{\ell} \triangleright_{e}^{s} q_{1}, \bar{v}_{1}$ and $\mathcal{B}^{\dagger}, q_{\ell} \triangleright_{e}^{s} q_{2}, \bar{v}_{2}$, then by induction hypothesis there exist $\bar{v}_{1}^{\prime} \geq \bar{v}_{1}$ and $\overline{\mathrm{v}}_{2}^{\prime} \geq \overline{\mathrm{v}}_{2}$ such that $\mathcal{B}^{\ddagger},\left(q_{\ell},[d]\right) \triangleright\left(q_{1}, s\right), \overline{\mathrm{v}}_{1}^{\prime}$ and $\mathcal{B}^{\ddagger},\left(q_{\ell},[d]\right) \triangleright\left(q_{2}, s\right), \overline{\mathrm{v}}_{2}^{\prime}$. Thanks to split ${ }^{\ddagger}$, this yields $\mathcal{B}^{\ddagger},(q \ell,[d]) \triangleright(q, s), \overline{\mathrm{v}}_{1}^{\prime}+\overline{\mathrm{v}}_{2}^{\prime}$ where $\overline{\mathrm{v}}_{1}^{\prime}+\overline{\mathrm{v}}_{2}^{\prime} \geq \overline{\mathrm{v}}_{1}+\overline{\mathrm{v}}_{2}$ as desired.

For the converse direction, given $s \subseteq[d]$ and $\overline{\mathrm{v}}$ in $\mathbb{N}^{d}$, we define $s \cdot \overline{\mathrm{v}}$ for each $0<i \leq d$ by

$$
(s \cdot \overline{\mathrm{v}})(i) \stackrel{\text { def }}{=} \begin{cases}\overline{\mathrm{v}}(i) & \text { if } i \in s, \\ 0 & \text { otherwise }\end{cases}
$$

Claim 4.5. If $\mathcal{B}^{\ddagger},\left(q_{\ell},[d]\right) \triangleright(q, s), \overline{\mathrm{v}}$, then $\mathcal{B}^{\dagger}, q_{\ell} \triangleright_{e} q, s \cdot \overline{\mathrm{v}}$.

Proof. We prove the claim by induction on the root judgement in $\mathcal{B}^{\ddagger}$. For the base case, $\mathcal{B}^{\ddagger},\left(q_{\ell},[d]\right) \triangleright\left(q_{\ell},[d]\right), \overline{0}$ indeed matches $\mathcal{B}^{\dagger}, q_{\ell} \triangleright_{e} q_{\ell},[d] \cdot \overline{0}$.

For the induction step, first assume that the judgement stems from incr and $\mathcal{B}^{\ddagger},\left(q_{\ell},[d]\right) \triangleright\left(q_{1}, s \cup\{i\}\right), \overline{\mathrm{v}}+\overline{\mathrm{e}}_{i}$. By induction hypothesis, $\mathcal{B}^{\dagger}, q_{\ell} \triangleright_{e}$ $q_{1},(s \cup\{i\}) \cdot\left(\overline{\mathrm{v}}+\overline{\mathrm{e}}_{i}\right)$. Two cases arise depending on whether $i \in s$. First suppose $i \in s$ : then $(s \cup\{i\}) \cdot\left(\overline{\mathrm{v}}+\overline{\mathrm{e}}_{i}\right)=s \cdot \overline{\mathrm{v}}+\overline{\mathrm{e}}_{i}$ and $q \stackrel{\overline{\mathrm{e}}_{i}}{\longrightarrow} q_{1}$ yields $\mathcal{B}^{\dagger}, q_{\ell} \triangleright_{e} q, s \cdot \overline{\mathrm{v}}$ as desired. Assuming $i \notin s$, let us decompose $\overline{\mathrm{v}}$ as $\overline{\mathrm{w}}+n \overline{\mathrm{e}}_{i}$ where $\overline{\mathrm{w}}(i)=0$ and $n \geq 0$ : then $(s \cup\{i\}) \cdot\left(\overline{\mathrm{v}}+\overline{\mathrm{e}}_{i}\right)=s \cdot \overline{\mathrm{w}}+(n+1) \overline{\mathrm{e}}_{i}$. We apply $n$ expansions on coordinate $i$ to show $\mathcal{B}^{\dagger}, q_{\ell} \triangleright_{e} q_{1}, s \cdot \overline{\mathrm{w}}+\overline{\mathrm{e}}_{i}$. Applying $q \stackrel{\overline{\mathrm{e}}_{i}}{\rightarrow} q_{1}$ then yields $\mathcal{B}^{\dagger}, q_{\ell} \triangleright_{e} q, s \cdot \overline{\mathrm{w}}$, where $s \cdot \overline{\mathrm{w}}=s \cdot \overline{\mathrm{v}}$ as desired.

Assume now that decr $\left.{ }^{\ddagger}\right)$ was applied to $\mathcal{B}^{\ddagger},\left(q_{\ell},[d]\right) \triangleright\left(q_{1}, s \cup\{i\}\right), \overline{\mathrm{v}}$. By induction hypothesis, $\mathcal{B}^{\dagger}, q_{\ell} \triangleright_{e} q_{1},(s \cup\{i\}) \cdot \overline{\mathrm{v}}$, and $q \stackrel{-\overline{\mathrm{e}}_{i}}{\longrightarrow} q_{1}$ yields $\mathcal{B}^{\dagger}, q_{\ell} \triangleright_{e} q,(s \cup\{i\}) \cdot \overline{\mathrm{v}}+\overline{\mathrm{e}}_{i}$, where $(s \cup\{i\}) \cdot \overline{\mathrm{v}}+\overline{\mathrm{e}}_{i}=(s \cup\{i\}) \cdot\left(\overline{\mathrm{v}}+\overline{\mathrm{e}}_{i}\right)$ as desired.

Finally, assume that split ${ }^{\ddagger}$ was applied to $\mathcal{B}^{\ddagger},\left(q_{\ell},[d]\right) \triangleright\left(q_{1}, s\right), \overline{\mathbf{v}}_{1}$ and to $\mathcal{B}^{\ddagger},\left(q_{\ell},[d]\right) \triangleright\left(q_{2}, s\right), \overline{\mathrm{v}}_{2}$. By induction hypothesis, $\mathcal{B}^{\dagger}, q_{\ell} \triangleright_{e} q_{1}, s \cdot \overline{\mathrm{v}}_{1}$ and $\mathcal{B}^{\dagger}, q_{\ell} \triangleright_{e} q_{2}, s \cdot \overline{\mathrm{v}}_{2}$, from which $q \rightarrow q_{1}+q_{2}$ yields $\mathcal{B}^{\dagger}, q_{\ell} \triangleright_{e} q,\left(s \cdot \overline{\mathrm{v}}_{1}\right)+\left(s \cdot \overline{\mathrm{v}}_{2}\right)$, where $\left(s \cdot \overline{\mathrm{v}}_{1}\right)+\left(s \cdot \overline{\mathrm{v}}_{2}\right)=s \cdot\left(\overline{\mathrm{v}}_{1}+\overline{\mathrm{v}}_{2}\right)$ as desired.

Proof of Proposition 4.3. If $\mathcal{B}, q_{\ell} \triangleright_{e} q_{r}, \overline{0}$, then by (1), $\mathcal{B}^{\dagger}, q_{\ell} \triangleright_{e}^{\emptyset} q_{r}, \overline{0}$, thus by Claim 4.4, there exists $\bar{v}$ such that $\mathcal{B}^{\ddagger},\left(q_{\ell},[d]\right) \triangleright\left(q_{r}, \emptyset\right), \bar{v}$, i.e. we can cover $\left(q_{r}, \emptyset\right)$ in $\mathcal{B}^{\ddagger}$. 
Conversely, if $\mathcal{B}^{\ddagger},\left(q_{\ell},[d]\right) \triangleright\left(q_{r}, \emptyset\right), \bar{v}$, then by Claim 4.5, $\mathcal{B}^{\dagger}, q_{\ell} \triangleright_{e} q_{r}, \emptyset \cdot \bar{v}$ where $\emptyset \cdot \bar{v}=\overline{0}$. Therefore $\mathcal{B}, q_{\ell} \triangleright_{e} q_{r}, \overline{0}$ in the original BVASS $\mathcal{B}$.

Theorem 4.6. Provability in $\mathbf{R}_{\rightarrow}$ is in 2-ExpTime.

Proof. By Proposition 4.1 and Proposition 4.3, from a provability instance $\langle F\rangle$, we can reduce to a coverability instance $\left\langle\mathcal{B}_{F}^{\ddagger},\left(q_{r}, \emptyset\right),\left(q_{\ell},[|F|]\right)\right\rangle$ where $\mathcal{B}_{F}^{\ddagger}$ has dimension $|F|$ and a number of states in $2^{p(|F|)}$ for a polynomial $p$. By Corollary 3.2, this coverability instance can be solved in double exponential time in $|F|$. Note that the coverability check can be performed on-the-fly from $F$ to avoid the explicit construction of $\mathcal{B}_{F}^{\ddagger}$.

\section{LOWER BOUND}

In this section, we exhibit a reduction from BVASS coverability to $\mathbf{R}_{\rightarrow}$ provability, thereby showing its 2-ExPTIme-hardness.

Previous reductions from counter machines to substructural logics in [16, [28, 15] actually reduce to provability in the logic extended with a theory encoding the rules of the system, which is then reduced to the basic logic. This last step relies in an essential way on the presence of exponential or additive connectives to 'dispose' of unused rules.

Having neither exponential nor additive connectives at our disposal, we introduce in Section 5.1 a comprehensive variant of the expansive reachability problem, where every rule should be employed at least once in the deduction. We further avoid the use of a theory and define in Section 5.2 a focusing calculus for $\mathbf{R}_{\rightarrow}$, from which the correctness of the reduction given in Section 5.3 will be facilitated.

5.1. Comprehensive Reachability. Given a BVASS $\mathcal{B}=\left\langle Q, d, T_{u}, T_{s}\right\rangle$ with expansive semantics and two states $q_{r}$ and $q_{\ell}$, the comprehensive reachability problem asks whether there exists a deduction tree of $\mathcal{B}$ with root label $\left(q_{r}, \overline{0}\right)$ and leaves label $\left(q_{\ell}, \overline{0}\right)$, such that every rule in $T_{u} \cup T_{s}$ is used at least once. In terms of root judgements, it asks whether $\mathcal{B}, T_{u} \cup T_{s}, q_{\ell} \triangleright_{e} q_{r}, \overline{0}$. We show that BVASS coverability can be reduced to comprehensive expansive reachability, hence by Fact 3.1:

Proposition 5.1. Comprehensive reachability in expansive ordinary BVASS is 2-ExPTIME-hard.

5.1.1. Increasing Reachability. Let us consider an instance $\left\langle\mathcal{B}, q_{r}, q_{\ell}\right\rangle$ of the coverability problem in an ordinary BVASS $\mathcal{B}=\left\langle Q, d, T_{u}, T_{s}\right\rangle$. As a first step, we construct an ordinary BVASS $\mathcal{B}^{\dagger} \stackrel{\text { def }}{=}\left\langle Q, d, T_{u}^{\dagger}, T_{s}\right\rangle$ with additional increases $q \stackrel{\overline{\mathrm{e}}_{i}}{\rightarrow} q$ for every $q$ in $Q$ and $0<i \leq d$. We claim that coverability in $\mathcal{B}$ is equivalent to reachability in $\mathcal{B}^{\dagger}$ :

Claim 5.2. There exists $\bar{v}$ in $\mathbb{N}^{d}$ such that $\mathcal{B}, q_{\ell} \triangleright q_{r}, \bar{v}$ if and only if $\mathcal{B}^{\dagger}, q_{\ell} \triangleright$ $q_{r}, \overline{0}$.

Proof Sketch. Clearly, if $\mathcal{B}, q_{\ell} \triangleright q_{r}, \overline{\mathrm{v}}$ for some $\overline{\mathrm{v}}$ in $\mathbb{N}^{d}$, then $\mathcal{B}^{\dagger}, q_{\ell} \triangleright q_{r}, \overline{\mathrm{v}}$, and using increases in $q_{r}$ shows $\mathcal{B}^{\dagger}, q_{\ell} \triangleright q_{r}, \overline{0}$. Conversely, if there is a reachability witness for $\left\langle\mathcal{B}^{\dagger}, q_{r}, q_{\ell}\right\rangle$, then we can assume that increases $q \stackrel{\overline{\mathrm{e}}_{i}}{\rightarrow} q$ occur as close to the root as possible. As increases occurring right below 


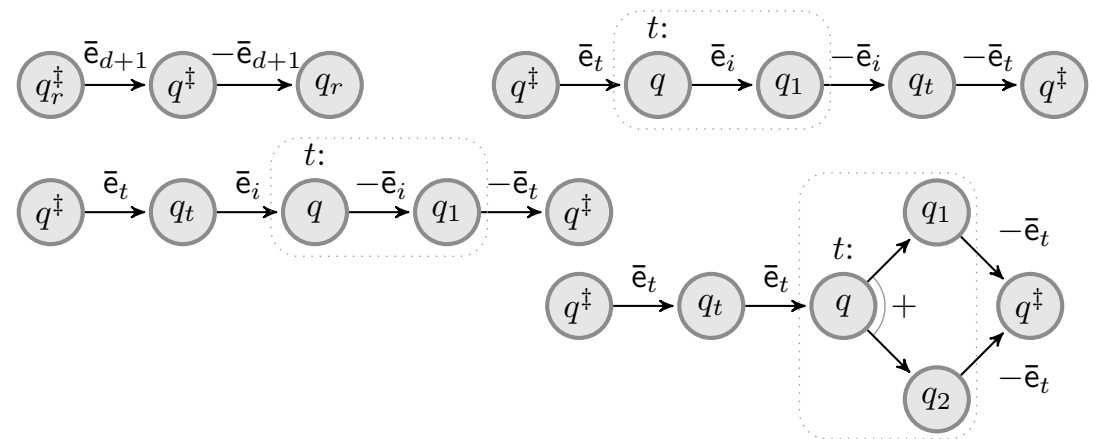

Figure 2. The rules of $\mathcal{B}^{\ddagger}$ in the proof of Proposition 5.1, where $t$ ranges over $T_{u}^{\dagger} \cup T_{s}$.

increments, decrements, or splits can be permuted locally to occur right above, such a reachability witness has all its increases at the root. The deduction tree below those increases is labelled $\left(q_{r}, \bar{v}\right)$ for some $\bar{v}$ in $\mathbb{N}^{d}$ and is also a deduction tree of $\mathcal{B}$.

5.1.2. Comprehensive Root Rules. The second step of the reduction from BVASS coverability builds an ordinary BVASS $\mathcal{B}^{\ddagger} \stackrel{\text { def }}{=}\left\langle Q^{\ddagger}, d+1+\right| T_{u}^{\dagger} \cup$ $T_{s}\left|, T_{u}^{\ddagger}, T_{s}\right\rangle$ where $Q^{\ddagger} \stackrel{\text { def }}{=} Q \uplus\left\{q^{\ddagger}, q_{r}^{\ddagger}\right\} \uplus\left\{q_{t} \mid t \in T_{u}^{\dagger} \cup T_{s}\right\}$. It features an additional set of unary 'root' rules - depicted in Figure 2 - designed to allow any rule in $T_{u}^{\ddagger} \cup T_{s}$ to be employed in a reachability witness.

The idea is to introduce a new state $q^{\ddagger}$ and a new coordinate for each rule $t$ in $T_{u}^{\dagger} \cup T_{s}$. Starting from $q^{\ddagger}, \mathcal{B}^{\ddagger}$ can simulate any rule $t$ from $T_{u}^{\dagger} \cup T_{s}$ by first incrementing by the corresponding unit vector $\overline{\mathrm{e}}_{t}$, then applying the rule, and finally decrementing by $\overline{\mathrm{e}}_{t}$ to return to $q^{\ddagger}$. This ensures that, if $\mathcal{B}^{\ddagger}, T, q_{\ell} \triangleright_{e} q^{\ddagger}, \overline{\mathrm{v}}$ for some $\overline{\mathrm{v}}$ in $\mathbb{N}^{d}$, then $\mathcal{B}^{\ddagger}, T^{\prime}, q_{\ell} \triangleright_{e} q^{\ddagger}, \overline{\mathrm{v}}$ where $T_{u}^{\dagger} \cup T_{s} \subseteq T^{\prime}$. The additional states and rules from $q_{r}^{\ddagger}$ and to $q_{r}$ then show that:

Claim 5.3. If $\mathcal{B}^{\dagger}, T, q_{\ell} \triangleright q_{r}, \overline{0}$ for some $T \subseteq T_{u}^{\dagger} \cup T_{s}$, then $\mathcal{B}^{\ddagger}, T_{u}^{\ddagger} \cup T_{s}, q_{\ell} \triangleright_{e} q_{r}^{\ddagger}, \overline{0}$.

Conversely, assume that $\mathcal{B}^{\ddagger}, q_{\ell} \triangleright_{e} q_{r}^{\ddagger}, \overline{0}$. This entails $\mathcal{B}^{\ddagger}, q_{\ell} \triangleright_{e} q^{\ddagger}, \overline{\mathrm{e}}_{d+1}$.

First assume that no decrement by $\overline{\mathrm{e}}_{t}$ for any $t$ in $T_{u}^{\dagger} \cup T_{s}$ is ever used in the corresponding expansive reachability witness. Then also no increment by $\overline{\mathrm{e}}_{t}$ occurs, and the only rule applicable at this point is $q^{\ddagger} \stackrel{-\overline{\mathrm{e}}_{d+1}}{\longrightarrow} q_{r}$. This yields a node $n$ labelled $\left(q_{r}, \overline{0}\right)$, and a deduction subtree rooted by $n$ where only rules of $\mathcal{B}^{\dagger}$ and expansions are applied. Because any expansion can be simulated in $\mathcal{B}^{\dagger}$ using an increase, this yields $\mathcal{B}^{\dagger}, q_{\ell} \triangleright q_{r}, \overline{0}$.

Assume now the opposite: there is at least one occurrence of a decrement by $\bar{e}_{t}$ in the expansive reachability witness. Consider the bottommost such occurrence along some branch, necessarily yielding a node with $q^{\ddagger}$ as state label. Then in the same way, below this bottommost occurrence, no increment by $\overline{\mathrm{e}}_{t}$ occurs, and the only rule applicable at this point is $q^{\ddagger} \stackrel{-\overline{\mathrm{e}}_{d+1}}{\longrightarrow} q_{r}$, which yields a node $n$ labelled $\left(q_{r}, \overline{\mathrm{v}}\right)$ for some $\overline{\mathrm{v}}$ in $\mathbb{N}^{d}$. The deduction subtree rooted by $n$ only uses rules of $\mathcal{B}^{\dagger}$ and expansions, thus as in the previous case $\mathcal{B}^{\dagger}, q_{\ell} \triangleright q_{r}, \bar{v}$. Using increases in $q_{r}$ then shows $\mathcal{B}^{\dagger}, q_{\ell} \triangleright q_{r}, \overline{0}$. Therefore, in all cases: 
Claim 5.4. If $\mathcal{B}^{\ddagger}, T_{u}^{\ddagger} \cup T_{s}, q_{\ell} \triangleright_{e} q_{r}^{\ddagger}, \overline{0}$, then $\mathcal{B}^{\dagger}, q_{\ell} \triangleright q_{r}, \overline{0}$.

By Claims 5.2, 5.3, and 5.4, $\mathcal{B}^{\ddagger}, T_{u}^{\ddagger} \cup T_{s}, q_{\ell} \triangleright_{e} q_{r}^{\ddagger}, \overline{0}$ if and only if there exists $\bar{v}$ in $\mathbb{N}^{d}$ such that $\mathcal{B}, q_{\ell} \triangleright q_{r}, \bar{v}$, thereby showing the correctness of our reduction.

5.2. Focusing Proofs in $\mathbf{R}_{\rightarrow}$. We enforce a particular proof policy in our simulation of BVASSs in $\mathbf{R}_{\rightarrow}$, which is inspired by the focusing proof techniques [2] employed to reduce non-determinism during proof search in sequent calculi. With only implication at our disposal, we find ourselves in a 'negative fragment', where focusing proofs have a very simple calculus $F \mathbf{R}_{\rightarrow}$. This is equivalent to restricting oneself to long normal forms in the associated $\lambda$-calculus.

A focusing sequent is of one of the two forms ' $\Gamma,[A] \Vdash B$ ' or ' $\Gamma \Vdash A$ ' where ' $[A]$ ' is called a focused formula. We let $\Gamma, \Delta, \ldots$ denote as before multisets of implicational formulæ and $A, B, C$ implicational formulæ. Here are the rules of the focusing calculus $F \mathbf{R}_{\rightarrow}$ :

$$
\begin{gathered}
\frac{\Gamma,[A] \Vdash a}{[a] \Vdash a} \text { (atomic) } \quad \frac{\Gamma,[\text { focus }) \quad \frac{\Gamma, A, A \Vdash a}{\Gamma, A \Vdash a}\left(\mathrm{C}^{f}\right)}{\Gamma, A \Vdash a} \\
\frac{\Gamma \Vdash A \quad \Delta,[B] \Vdash a}{\Gamma, \Delta,[A \rightarrow B] \Vdash a}\left(\rightarrow \rightarrow_{\mathrm{L}}^{f}\right) \quad \frac{\Gamma, A \Vdash B}{\Gamma \Vdash A \rightarrow B}\left(\rightarrow \rightarrow_{\mathrm{R}}^{f}\right)
\end{gathered}
$$

Note that our focusing calculus $F \mathbf{R}_{\rightarrow}$ gives the priority to right implications $\rightarrow \rightarrow_{R}^{f}$ ) over the left implications $\rightarrow \rightarrow_{\mathrm{L}}^{f}$, focus (focus), and contractions $\left(C^{f}\right)$ : the latter can only be applied to sequents with atomic succedents $a$ in $\mathcal{\mathcal { A }}$. A similar observation is that a focusing sequent $\Gamma,[a] \Vdash A$ is provable if and only if $A=a$ is atomic and $\Gamma=\emptyset$ is the empty multiset, since (atomic is the only rule yielding a sequent with a focused atomic formula $[a]$.

Theorem $5.5\left(F \mathbf{R}_{\rightarrow}\right.$ is sound and complete). A sequent $\Gamma \vdash A$ is provable in $L \mathbf{R}_{\rightarrow}$ if and only if the focusing sequent $\Gamma \Vdash A$ is provable in $F \mathbf{R}_{\rightarrow}$.

We prove Theorem 5.5 in Appendix A, using the admissibility of a suitable cut rule in $F \mathbf{R}_{\rightarrow}$.

5.3. From Comprehensive Expansive Reachability to $\boldsymbol{F} \mathbf{R}_{\rightarrow}$. Let us consider a comprehensive expansive reachability instance $\left\langle\mathcal{B}, q_{r}, q_{\ell}\right\rangle$ where $\mathcal{B}=\left\langle Q, d, T_{u}, T_{s}\right\rangle$. We are going to construct an implicational formula $F$ such that $\vdash F$ if and only if $\mathcal{B}, T_{u} \cup T_{s}, q_{\ell} \triangleright_{e} q_{r}, \overline{0}$.

We work for this on the set of atomic formulæ $Q \uplus\left\{e_{i} \mid 0<i \leq d\right\}$, and associate to a root judgement $\mathcal{B}, T, q_{\ell} \triangleright_{e} q, \bar{v}$ a focusing sequent

$$
q_{\ell}, \Delta_{T}, \Gamma_{\bar{v}} \Vdash q
$$

where $\Delta_{T}$ encodes the rules in $T \subseteq T_{u} \cup T_{s}$ and $\Gamma_{\bar{v}}$ encodes $\bar{v}$ : let $T=$ $\left\{t_{1}, \ldots, t_{k}\right\}$ and $\overline{\mathrm{v}}=c_{1} \overline{\mathrm{e}}_{1}+\cdots+c_{d} \overline{\mathrm{e}}_{d}$, then

$$
\begin{gathered}
\Delta_{T} \stackrel{\text { def }}{=}\left\ulcorner t_{1}\right\urcorner, \ldots,\left\ulcorner t_{k}\right\urcorner, \\
\Gamma_{\overline{\mathrm{v}}} \stackrel{\text { def }}{=} e_{1}^{c_{1}}, \ldots, e_{d}^{c_{d}},
\end{gathered}
$$


where ' $\ulcorner t\urcorner$ ' is the individual encoding of rule $t$ and ' $A$ ' ' stands for $c$ repetitions of the formula $A$. We use the following individual rule encodings:

$$
\begin{array}{r}
\left\ulcorner q \stackrel{\overline{\mathrm{e}}_{i}}{\rightarrow} q_{1}\right\urcorner \stackrel{\text { def }}{=}\left(e_{i} \rightarrow q_{1}\right) \rightarrow q, \\
\left\ulcorner q \stackrel{-\overline{\mathrm{e}}_{i}}{\longrightarrow} q_{1}\right\urcorner \stackrel{\text { def }}{=} q_{1} \rightarrow\left(e_{i} \rightarrow q\right), \\
\left\ulcorner q \rightarrow q_{1}+q_{2}\right\urcorner \stackrel{\text { def }}{=} q_{1} \rightarrow\left(q_{2} \rightarrow q\right) .
\end{array}
$$

Then proof search in $F \mathbf{R}_{\rightarrow}$ is easily seen to implement deductions in $\mathcal{B}$ :

Claim 5.6 (Completeness). If $\mathcal{B}, T, q_{\ell} \triangleright_{e} q, \bar{v}$, then $q_{\ell}, \Delta_{T}, \Gamma_{\overline{\mathrm{v}}} \Vdash q$.

Proof. We proceed by induction on the structure of the root judgement. For the base case, i.e. for $\mathcal{B}, \emptyset, q_{\ell} \triangleright_{e} q_{\ell}, \overline{0}$, we have

$$
\frac{\left[q_{\ell}\right] \Vdash q_{\ell}}{q_{\ell} \Vdash q_{\ell}} \text { atomic }
$$

as desired.

For the induction step, if the last applied rule is an increment $t=q \stackrel{\overline{\mathrm{e}}_{i}}{\rightarrow} q_{1}$ on a judgement $\mathcal{B}, T, q_{\ell} \triangleright_{e} q_{1}, \overline{\mathrm{v}}+\overline{\mathrm{e}}_{i}$, then

i.h.

$$
\frac{\frac{q_{\ell}, \Delta_{T}, \Gamma_{\overline{\mathrm{v}}}, e_{i} \Vdash q_{1}}{q_{\ell}, \Delta_{T}, \Gamma_{\overline{\mathrm{v}}} \Vdash e_{i} \rightarrow q_{1}} \stackrel{\left.G_{\mathrm{R}}^{f}\right]}{[q] \Vdash q} \text { atomic }}{\frac{q_{\ell}, \Delta_{T}, \Gamma_{\overline{\mathrm{v}}},\left[\left(e_{1} \rightarrow q_{1}\right) \rightarrow q\right] \Vdash q}{q_{\ell}, \Delta_{T}, \Gamma_{\overline{\mathrm{v}}},\left(e_{1} \rightarrow q_{1}\right) \rightarrow q \Vdash q}}
$$

and an additional contraction $\mathrm{C}^{f}$ if $t \in T$ shows $q_{\ell}, \Delta_{T \cup\{t\}}, \Gamma_{\overline{\mathrm{v}}} \Vdash q$ as desired.

If the last applied rule is a decrement $t=q \stackrel{-\overline{\mathrm{e}}_{i}}{\longrightarrow} q_{1}$ on a judgement $\mathcal{B}, T, q_{\ell} \triangleright_{e} q_{1}, \bar{v}$, then

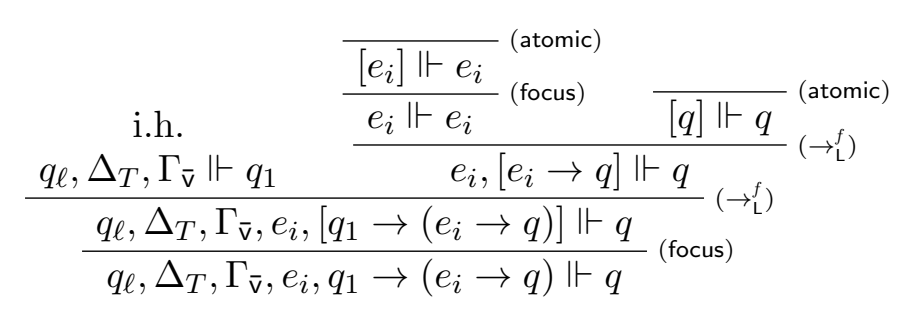

and a contraction $\left[\mathrm{C}^{f}\right]$ if $t \in T$ shows $q_{\ell}, \Delta_{T \cup\{t\}}, \Gamma_{\overline{\mathrm{v}}+\overline{\mathrm{e}}_{i}} \Vdash q$ as desired.

If the last applied rule is an expansion on a judgement $\mathcal{B}, T, q_{\ell} \triangleright_{e} q, \overline{\mathrm{v}}+2 \overline{\mathrm{e}}_{i}$, then

i.h.

$$
\frac{q_{\ell}, \Delta_{T}, \Gamma_{\overline{\mathrm{v}}}, e_{i}, e_{i} \Vdash q}{q_{\ell}, \Delta_{T}, \Gamma_{\overline{\mathrm{v}}}, e_{i} \Vdash q}
$$

as desired.

Finally, if the last applied rule is a split $t=q \rightarrow q_{1}+q_{2}$ on two judgements $\mathcal{B}, T_{1}, q_{\ell} \triangleright_{e} q_{1}, \bar{v}_{1}$ and $\mathcal{B}, T_{2}, q_{\ell} \triangleright_{e} q_{2}, \bar{v}_{2}$, then 


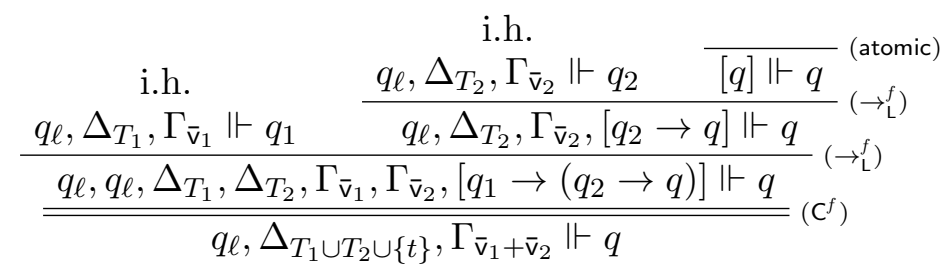

as desired.

The interest of the focusing calculus $F \mathbf{R}_{\rightarrow}$ is that, starting from a sequent $q_{\ell}, \Delta, \Gamma,[\ulcorner t\urcorner] \Vdash q$ where $\Delta$ is in $\mathbb{N}^{\Delta_{T u} \cup T_{s}}$ and $\Gamma$ in $\mathbb{N}^{\left\{e_{i} \mid 0<i \leq d\right\}}$ and the focus is on the encoding of a rule $t$, there is no choice but to follow the proof trees shown in the proof of Claim 5.6. Given a multiset $m$ in $\mathbb{N}^{E}$ for some set $E$, we write

$$
\sigma(m) \stackrel{\text { def }}{=}\{e \in E \mid m(e)>0\}
$$

for the support of $m$.

Claim 5.7 (Soundness). Let $\Delta$ be in $\mathbb{N}^{\Delta_{T_{u}} \cup T_{s}}, \Gamma$ in $\mathbb{N}^{\left\{e_{i} \mid 0<i \leq d\right\}}, q$ in $Q$, and $n>0$. Then $q_{\ell}^{n}, \Delta, \Gamma \Vdash q$ implies $\mathcal{B}, \sigma(\Delta), q_{\ell} \triangleright_{e} q, \overline{\mathrm{v}}_{\Gamma}$.

Proof. Note that $n=0$ would yield an unprovable sequent. We proceed by induction on the structure of a proof tree for the focusing sequent. The only applicable rules in a proof search from $q_{\ell}^{n}, \Delta, \Gamma \Vdash q$ are focus and ( $C^{f}$ ). In the latter case, we distinguish three cases depending on the contracted formula $A$ :

- If $A=q_{\ell}$, i.e. if $q_{\ell}^{n+1}, \Delta, \Gamma \Vdash q$, then by induction hypothesis $\mathcal{B}, \sigma(\Delta), q_{\ell} \triangleright_{e} q, \bar{v}_{\Gamma}$ as desired.

- If $A=e_{i}$ in $\Gamma$, then by induction hypothesis $\mathcal{B}, \sigma(\Delta), q_{\ell} \triangleright_{e} q, \overline{\mathrm{v}}_{\Gamma}+\overline{\mathrm{e}}_{i}$, and an expansion yields $\mathcal{B}, \sigma(\Delta), q_{\ell} \triangleright_{e} q, \bar{v}_{\Gamma}$ as desired.

- If $A=\ulcorner t\urcorner$ for some rule $t$ in $\Delta$, then the support $\sigma(\Delta)$ is not changed and by induction hypothesis $\mathcal{B}, \sigma(\Delta), q_{\ell} \triangleright_{e} q, \bar{v}_{\Gamma}$ as desired.

In the former case, we also distinguish three cases depending on which formula $A$ receives the focus:

- If $A=q_{\ell}$, necessarily $\Delta$ and $\Gamma$ are empty and $q=q_{\ell}$, and indeed $\mathcal{B}, \emptyset, q_{\ell} \triangleright_{e} q_{\ell}, \overline{0}$.

- If $A=e_{i}$ in $\Gamma$, then proof search fails since $q \neq e_{i}$.

- If $A=\ulcorner t\urcorner$ in $\Delta$, then proof search needs to follow the proof trees used in the proof of Claim 5.6, and applying the induction hypothesis on the open leaves of these trees allows to conclude in each case.

Theorem 5.8. Provability in $\mathbf{R}_{\rightarrow}$ is 2-ExPTIME-hard.

Proof. We reduce from the comprehensive expansive reachability problem, which is 2-ExPTIme-hard by Proposition 5.1. From an instance $\left\langle\mathcal{B}, q_{r}, q_{\ell}\right\rangle$ where $\mathcal{B}=\left\langle Q, d, T_{u}, T_{s}\right\rangle$, we construct a formula $F \stackrel{\text { def }}{=} q_{\ell} \rightarrow \varphi\left(T_{u} \cup T_{s}, q_{r}\right)$ defined by

$$
\varphi\left(\emptyset, q_{r}\right) \stackrel{\text { def }}{=} q_{r}, \quad \varphi\left(T \uplus\{t\}, q_{r}\right) \stackrel{\text { def }}{=}\ulcorner t\urcorner \rightarrow \varphi\left(T, q_{r}\right) .
$$

By Theorem 5.5, $\vdash F$ if and only if $\Vdash F$. The latter holds if and only if $q_{\ell}, \Delta_{T_{u} \cup T_{s}} \Vdash q_{r}$ since we can only apply $\rightarrow \rightarrow_{\mathrm{R}}^{f}$. Then this occurs if and only if $\mathcal{B}, T_{u} \cup T_{s}, q_{\ell} \triangleright_{e} q_{r}, \overline{0}$ by Claim 5.6 and Claim 5.7. 

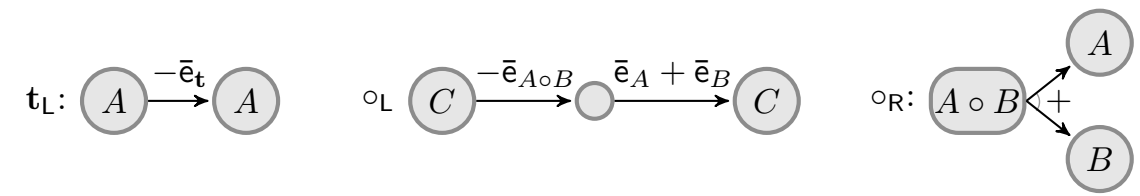

Figure 3. The additional BVASS rules for $L \mathbf{R}_{\rightarrow, \circ}^{\mathbf{t}}$.

\section{Extensions}

The complexity bounds proven in Theorem 4.6 and Theorem 5.8 for $\mathbf{R}_{\rightarrow}$ can be generalised to larger fragments of $\mathbf{R}$ and of propositional intuitionistic contractive linear logic (ILLC).

6.1. Adding Multiplicatives. The sequent system $L \mathbf{R}_{\rightarrow}$ for $\mathbf{R}_{\rightarrow}$ can be extended to accommodate further multiplicative connectives: the fusion connective $\circ$ (aka. 'co-tenability' in [1]) and the sentential constant t:

$$
\begin{array}{lc}
\frac{\Gamma \vdash A}{\Gamma, \mathbf{t} \vdash A}\left(\mathbf{t}_{\mathrm{L}}\right) & \left.\frac{\vdash \mathbf{t}}{\mathbf{t}_{\mathrm{R}}}\right) \\
\frac{\Gamma, A, B \vdash C}{\Gamma, A \circ B \vdash C}\left(\mathrm{o}_{\mathrm{L}}\right) & \frac{\Gamma \vdash A \quad \Delta \vdash B}{\Gamma, \Delta \vdash A \circ B}\left(\mathrm{\circ}_{\mathrm{R}}\right)
\end{array}
$$

Let us call $L \mathbf{R}_{\rightarrow, 0}^{\mathrm{t}}$ the resulting sequent system. The BVASS $\mathcal{B}_{F}$ presented in Section 4.2 can be extended in a straightforward manner with the rules of Figure 3 and by identifying $q_{\ell}$ with $\mathbf{t}$. Thanks to Proposition 4.3 and Corollary 3.2, this shows that provability in $L \mathbf{R}_{\rightarrow, 0}^{\mathbf{t}}$ is in 2-EXPTIME.

Note that the sequent system $L \mathbf{R}_{\rightarrow, 0}^{\mathbf{t}}$ is the same as that of intuitionistic multiplicative contractive linear logic (IMLLC), where $\rightarrow$, $\circ$, and $\mathbf{t}$ are usually noted respectively $\multimap, \otimes$, and $\mathbf{1}$.

6.2. Adding Exponentials. In fact, essentially the same reduction from sequent calculus to expansive BVASS reachability can be carried over for the more general multiplicative exponential fragment of intuitionistic contractive linear logic with bottom IMELZC, see [15, Proposition 9]. The main differences are that:

(1) The exponential connectives incur an exponential blow-up in the number of states of the constructed BVASS $\mathcal{B}_{F}$ : its state space now contains $S \times 2^{S !}$ where $S$ ! is the set of exponential subformulæ of $F$. The subsequent reduction to coverability in Section 4.3 then performs a product with $2^{S \backslash S !}$ (contractions in exponential subformulæ being already handled), hence the resulting state space remains of size $2^{p(|F|)}$ for some polynomial $p$.

(2) The exponential connectives also require an additional operation of full zero test: as shown in [15, Lemma 3], this operation can be eliminated at no cost in complexity.

By Theorem 5.8, Proposition 4.3, and Corollary 3.2, we conclude:

Theorem 6.1. Provability in the logics $\mathbf{R}_{\rightarrow}, \mathbf{R}_{\rightarrow}^{\mathbf{t}}$, IMLLC, IMELLC, and IMELZC is 2-EXPTIME-complete. 
It seems likely that the non-intuitionistic variants MLLC and MELLC, i.e. multiplicative and multiplicative exponential contractive linear logic, are also 2-ExpTIME-complete: the upper bound follows from the bound on IMELZC, and the lower bound should not cause any difficulty, but is beyond the scope of this paper.

6.3. Adding Additives. The key difference between $L \mathbf{R}_{\rightarrow, 0}^{\mathbf{t}}$ and the AcKERMANN-complete sequent calculus studied by Urquhart [28] for the conjunctiveimplicational fragment $\mathbf{R}_{\rightarrow, \wedge}$ is the absence of conjunction $\wedge$ and disjunction $\checkmark$, which are additive connectives that require a richer model of alternating BVASS; see [15].

\section{Concluding Remarks}

Besides closing a longstanding open problem, the proof that $\mathbf{R}_{\rightarrow}$ is 2ExPTIME-complete paves the way for new investigations:

- In spite of the high worst-case complexity of BVASS coverability, Majumdar and Wang [17] have recently presented a practical algorithm with encouraging initial results. The reduction in Section 4 allows to transfer their techniques to $\mathbf{R}_{\rightarrow}$ provability, but might incur a worst-case exponential blow-up.

- Provability in the related implicational fragment $\mathbf{T}_{\rightarrow}$ of ticket entailment has recently been proven decidable independently by Padovani [20] and Bimbó and Dunn [3]. Although the complexity of this problem is currently unknown, the latter proof relies on provability in $L \mathbf{R}_{\rightarrow}^{\mathbf{t}}$, which we prove to be 2-ExPTIME-complete in Section 6 .

Acknowledgements. The author thanks David Baelde for his excellent suggestion of employing focusing proofs and helpful discussions around their uses.

\section{Appendix A. Focusing Calculus}

The purpose of this appendix is to prove Theorem 5.5. $F \mathbf{R}_{\rightarrow}$ is sound and complete for $\mathbf{R}_{\rightarrow}$. We first prove some preliminary statements:

Lemma A.1 (Right implication is invertible). The sequent $\Gamma \Vdash A \rightarrow B$ is provable in $F \mathbf{R}_{\rightarrow}$ if and only if $\Gamma, A \Vdash B$ is provable.

Proof. No other rule of $F \mathbf{R}_{\rightarrow}$ can yield a non-atomic succedent $A \rightarrow B$.

Lemma A.2 (Identity is admissible). Let $A=A_{n} \rightarrow \cdots \rightarrow A_{1} \rightarrow$ a where the $A_{i}$ 's are arbitrary implicational formula and $a$ is atomic. Then the following rules are admissible in $F \mathbf{R}_{\rightarrow}$ :

$$
\overline{A_{1}, \ldots, A_{n},[A] \Vdash a}\left(\operatorname{Id}_{[]}\right) \quad \overline{A \Vdash A}\left(\operatorname{ld}^{f}\right)
$$

Proof. Observe that $\left(\mathrm{Id}^{f}\right)$ can always be derived from $\left[\mathrm{Id}_{[]}\right]$by

$$
\frac{\frac{A_{1}, \ldots, A_{n},[A] \Vdash a}{A_{1}, \ldots, A_{n}, A \Vdash a}}{A \Vdash A} \rightarrow \rightarrow_{\mathrm{R}}^{f}
$$


We thus prove the two rules simultaneously by induction over the structure of $A$. The base case for $A$ atomic (i.e. for $n=0$ ) holds thanks to the atomic rule; for the induction step, we have the proof

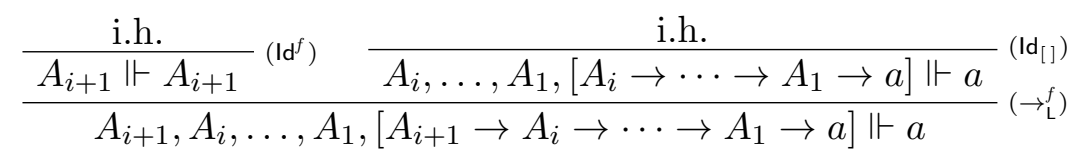

Let us introduce extra notation: lower-case $\gamma, \delta, \ldots$ denote multisets of implicational or focused formulæ containing at most one focused formula.

Lemma A.3 (Mix is admissible). The mix rules (mix) and $\left(\operatorname{mix}_{[]}\right.$are admissible in $F \mathbf{R} \rightarrow$ for all $n \geq 0$ :

$$
\frac{\Gamma \Vdash A \quad \delta, A^{n+1} \Vdash a}{\Gamma, \delta \Vdash a}(\operatorname{mix}) \quad \frac{\Gamma \Vdash A \quad \Delta, A^{n},[A] \Vdash a}{\Gamma, \Delta \Vdash a}\left(\operatorname{mix}_{[]}\right)
$$

Proof. Assume that the two premises of a mix rule can be proved in $F \mathbf{R}_{\rightarrow}$. We show by induction, first on the structure of the cut formula $A$, and second on the structure of the proof of the right premise, that the conclusion of the mix rule can be proved in $F \mathbf{R}_{\rightarrow}$. Let us consider for this the last rule applied in the proof of the right premise:

atomic identity; this is only possible for

$$
\frac{\Gamma \Vdash a \quad \overline{[a] \Vdash a}}{\Gamma \Vdash a} \stackrel{\text { atomic }}{\text { mix } \left.]_{[}\right]}
$$

and could be obtained directly from the left premise.

focus: this is only possible for

$$
\frac{\Gamma \Vdash A \frac{\Delta, A^{n},[A] \Vdash a}{\Delta, A^{n+1} \Vdash a} \text { focus }}{\Gamma, \Delta \Vdash a}
$$

and could be obtained directly from $\left(\operatorname{mix}_{[]}\right]$with a sub-proof of its right premise, thus by induction hypothesis there is a mix-free proof of $\Gamma, \Delta \Vdash a$.

contraction: this is only possible for

$$
\frac{\Gamma \Vdash A \quad \frac{\Delta, A^{n+2} \Vdash a}{\Delta, A^{n+1} \Vdash a} \text { 兵 }}{\Gamma, \Delta \Vdash a}
$$

and could be obtained directly from mix with a sub-proof of its right premise, thus by induction hypothesis there is a mix-free proof of $\Gamma, \Delta \Vdash a$.

left implication: here we consider two cases depending on whether (mix) or $\left(\operatorname{mix}_{[]}\right)$is applied. Here is the situation with (mix):

$$
\frac{\Gamma \Vdash A \quad \frac{\Delta_{1}, A^{n_{1}} \Vdash B \quad \Delta_{2}, A^{n_{2}},[C] \Vdash a}{\Delta_{1}, \Delta_{2}, A^{n_{1}+n_{2}},[B \rightarrow C] \Vdash a}}{\Gamma, \Delta_{1}, \Delta_{2},[B \rightarrow C] \Vdash a} \text { mix }
$$

We replace this proof by the following: 


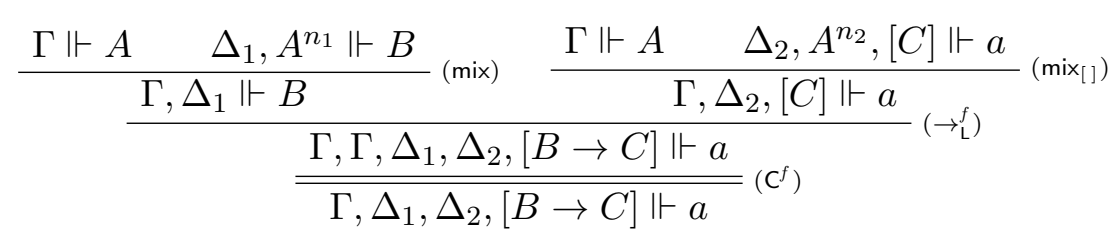

where the two introduced mix rules have sub-proofs of the original right premise as right premises, and can therefore be turned into mix-free proofs by induction hypothesis.

The situation with $\left(\operatorname{mix}_{[]}\right]$is more involved:

$$
\frac{\Gamma \Vdash A \rightarrow B \quad \frac{\Delta_{1},(A \rightarrow B)^{n_{1}} \Vdash A \quad \Delta_{2},(A \rightarrow B)^{n_{2}},[B] \Vdash a}{\Delta_{1}, \Delta_{2},(A \rightarrow B)^{n_{1}+n_{2}},[A \rightarrow B] \Vdash a}}{\Gamma, \Delta_{1}, \Delta_{2} \Vdash a}\left[\rightarrow_{L}^{f}\right]
$$

We prove this in two steps: we first prove $\Gamma, \Delta_{2}, A \Vdash a$ by

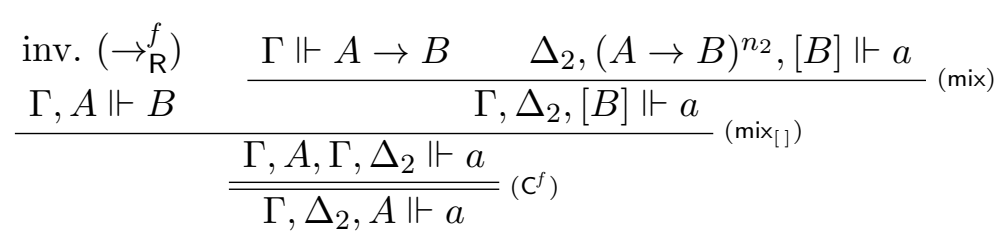

This relies on Lemma A.1 to show $\Gamma, A \Vdash B$. By induction hypothesis, the top (mix) is applied to a sub-proof of the right premise and the bottom $\left(\operatorname{mix}_{[]}\right)$uses a subformula $B$ of the original cut formula $A \rightarrow B$, thus both have mix-free proofs.

As a second step, we use $\Gamma, \Delta_{2}, A \Vdash a$ in

$$
\begin{gathered}
\Gamma \Vdash A \rightarrow B \quad \Delta_{1},(A \rightarrow B)^{n_{1}} \Vdash A \\
\frac{\Gamma, \Delta_{1} \Vdash A}{\frac{\Gamma, \Gamma, \Delta_{1}, \Delta_{2} \Vdash a}{\Gamma, \Delta_{1}, \Delta_{2} \Vdash a} \Gamma, \Delta_{2}, A \Vdash a} \text { [mix }
\end{gathered}
$$

The top (mix) has a sub-proof as right premise, while the bottom (mix) uses a subformula $A$ of the cut formula $A \rightarrow B$, thus both have mix-free proofs, yielding an overall mix-free proof for $\Gamma, \Delta_{1}, \Delta_{2} \Vdash a$.

right implication: cannot yield a sequent with an atomic succedent $a$.

Theorem 5.5 ( $F \mathbf{R}_{\rightarrow}$ is sound and complete). A sequent $\Gamma \vdash A$ is provable in $L \mathbf{R}_{\rightarrow}$ if and only if the focusing sequent $\Gamma \Vdash A$ is provable in $F \mathbf{R}_{\rightarrow}$.

Proof. First observe that any proof in $F \mathbf{R}_{\rightarrow}$ can be transformed into a proof in $L \mathbf{R}_{\rightarrow}$ by removing the focus information in sequents and suppressing (focus rules.

Conversely, we show by induction over the structure of the proof of $\Gamma \vdash A$ in $L \mathbf{R}_{\rightarrow}$ that $\Gamma \Vdash A$ in $F \mathbf{R}_{\rightarrow}$. To this end, let us examine the last applied rule in the proof of $\Gamma \vdash A$ :

identity; by Lemma A.2, the corresponding sequent $A \Vdash A$ can be proved in $F \mathbf{R}_{\rightarrow}$.

contraction; let $B=B_{1} \rightarrow \cdots \rightarrow B_{m} \rightarrow b$ where the $B_{i}$ 's are implicational formulæ and $b$ is an atomic formula. By induction hypothesis, $\Gamma, A, A \Vdash B$ is provable, thus by Lemma A.1, we have the proof 


$$
\frac{\frac{\Gamma, A, A, B_{1}, \ldots, B_{m} \Vdash b}{\Gamma, A, B_{1}, \ldots, B_{m} \Vdash b}}{\Gamma, A \Vdash B} \rightarrow_{\mathrm{C}^{f}}^{f}
$$

left implication: by induction hypothesis, $\Gamma \Vdash A$ and $\Delta, B \Vdash C$. Let $B=B_{1} \rightarrow \cdots \rightarrow B_{m} \rightarrow b$ and $C=C_{1} \rightarrow \cdots \rightarrow C_{n} \rightarrow c$ where $b$ and $c$ are atomic. Using Lemma A.2 and Lemma A.3, we first prove

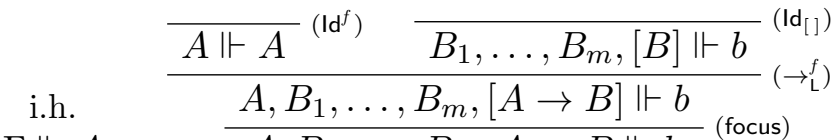

$$
\begin{aligned}
& \frac{\Gamma \Vdash A}{\frac{\Gamma, B_{1}, \ldots, B_{m}, A \rightarrow B \Vdash b}{\Gamma, A \rightarrow B \Vdash B}} \underset{\mathrm{R}}{\frac{f}{\mathrm{f}}} \text { mix }
\end{aligned}
$$

Thus, using Lemma A.1 and Lemma A.3, we have:

$$
\begin{aligned}
& \text { i.h. and inv. } \rightarrow_{\mathrm{R}}^{f} \\
& \frac{\Gamma, A \rightarrow B \Vdash B \quad \Delta, B, C_{1}, \ldots, C_{n} \Vdash c}{\frac{\Gamma, \Delta, A \rightarrow B, C_{1}, \ldots, C_{n} \Vdash c}{\Gamma, \Delta, A \rightarrow B \Vdash C}}
\end{aligned}
$$

right implication; the $F \mathbf{R}_{\rightarrow}$ rule $\rightarrow_{\mathrm{R}}^{f}$ ) is identical.

\section{Appendix B. Branching Vector Addition Systems}

Our goal in this appendix is to prove Corollary 3.2. Let us first define branching VASs in a more formal way. A BVAS is a tuple $\mathcal{B}=\left\langle d, T_{u}, T_{s}\right\rangle$ where both $T_{u}$ and $T_{s}$ are finite sets of rules in $\mathbb{Z}^{d}$. The configurations of $\mathcal{B}$ are thus in $\mathbb{N}^{d}$, and deductions follow the rules:

$$
\frac{\bar{u}+\bar{v}}{\bar{v}}\left(\text { unary }^{\prime}\right) \quad \frac{\bar{u}+\bar{v}_{1}+\bar{v}_{2}}{\bar{v}_{1} \quad \bar{v}_{2}}\left(\text { split }^{\prime}\right)
$$

for $\overline{\mathrm{u}}$ in $T_{u}$, respectively $T_{s}$.

The coverability problem then takes as input a BVAS $\mathcal{B}$ of dimension $d$, a root vector $\overline{\mathrm{v}}_{r}$, and a leaf vector $\overline{\mathrm{v}}_{\ell}$, both in $\mathbb{N}^{d}$, and asks for the existence of a deduction tree using (unary') and split') with root labelled by some $\overline{\mathrm{v}} \geq \overline{\mathrm{v}}_{r}$ for the product ordering over $\mathbb{N}^{d}$ and leaves labelled by $\overline{\mathrm{v}}_{\ell}$. Defining root judgements $\mathcal{B}, \bar{v}_{\ell} \triangleright \bar{v}$ in the natural way, coverability then asks whether there exists $\bar{v} \geq \bar{v}_{r}$ such that $\mathcal{B}, \bar{v}_{\ell} \triangleright \bar{v}$.

B.1. From BVAS to BVASS. As mentioned earlier, BVAS coverability is easy to reduce to BVASS coverability. Given a BVAS coverability instance $\left\langle\mathcal{B}, \bar{v}_{r}, \bar{v}_{\ell}\right\rangle$ with $\mathcal{B}=\left\langle d, T_{u}, T_{s}\right\rangle$, we build a BVASS coverability instance $\left\langle\mathcal{B}^{\prime}, q_{r}, q_{\ell}\right\rangle$ where $\mathcal{B}^{\prime} \stackrel{\text { def }}{=}\left\langle Q, d, T_{u}^{\prime}, T_{s}^{\prime}\right\rangle$ is equipped with a dummy state $q$, one state $q_{\overline{\mathrm{u}}}$ for each split rule $\overline{\mathrm{u}}$ in $T_{s}$, and two states $q_{r}$ and $q_{\ell}$. Unary rules $\overline{\mathrm{u}}$ in $T_{u}$ are encoded as $q \stackrel{-\overline{\mathrm{u}}}{\longrightarrow} q$. Split rules $\overline{\mathrm{u}}$ in $T_{s}$ are encoded as $q \stackrel{-\overline{\mathrm{u}}}{\longrightarrow} q_{\overline{\mathrm{u}}} \rightarrow q+q$. The root condition is checked by a rule $q_{r} \stackrel{\overline{\mathrm{v}}_{r}}{\longrightarrow} q$, the leaf condition by a rule $q \stackrel{-\bar{v}_{\ell}}{\longrightarrow} q_{\ell}$. Then a deduction tree with root $\bar{v}$ and leaves $\bar{v}_{\ell}$ exists for $\mathcal{B}$ if and only if a deduction tree with root $(q, \bar{v})$ and leaves $\left(q, \bar{v}_{\ell}\right)$ exists for $\mathcal{B}^{\prime}$, and the result follows. 
B.2. From BVASS to BVAS. The converse reduction is more involved. Demri et al. sketch in [10] a reduction that incurs a linear increase in the dimension, but we need here to be more economical.

Given a vector $\overline{\mathrm{v}}$ in $\mathbb{N}^{d}$, we write $\|\overline{\mathrm{v}}\|$ for its infinite norm $\max _{0<i \leq d} \overline{\mathrm{v}}(i)$. For a set of vectors $T,\|T\|$ then denotes $\max _{\bar{v} \in T}\|\bar{v}\|$. When considering BVAS(S) coverability problems, we assume a binary encoding of vectors, e.g. $\left\|T_{u}\right\| \in 2^{O(|\mathcal{B}|)}$.

Corollary 3.2. Coverability in a $B V A S S \mathcal{B}=\left\langle Q, d, T_{u}, T_{s}\right\rangle$ can be solved

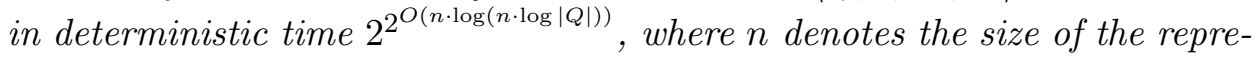
sentation of $\left\langle d, T_{u}\right\rangle$.

Proof. Let us consider an instance $\left\langle\mathcal{B}, q_{r}, q_{\ell}\right\rangle$ of the BVASS coverability problem, where $\mathcal{B}=\left\langle Q, d, T_{u}, T_{s}\right\rangle$. We construct a BVAS coverability instance $\left\langle\mathcal{B}^{\prime}, \overline{\mathrm{v}}_{r}, \overline{\mathrm{v}}_{\ell}\right\rangle$ where $\mathcal{B}^{\prime} \stackrel{\text { def }}{=}\left\langle 6+d, T_{u}^{\prime}, T_{s}^{\prime}\right\rangle$. We do not assume $\mathcal{B}$ to be ordinary, i.e. we have the following general rule for root judgements derived from unary rules in $T_{u}$ :

$$
\frac{\mathcal{B}, T, q_{\ell} \triangleright q_{1}, \overline{\mathrm{v}}+\overline{\mathrm{u}}}{\mathcal{B}, T \cup\left\{q \stackrel{\overline{\mathrm{u}}}{\rightarrow} q_{1}\right\}, q_{\ell} \triangleright q, \overline{\mathrm{v}}}
$$

The idea of the reduction is to encode each state from the finite set $Q=$ $\left\{q_{0}, \ldots, q_{|Q|-1}\right\}$ using two extra dimensions. For a state $q_{i}$ with $0 \leq i<|Q|$, we write $\left\ulcorner q_{i}\right\urcorner$ for the pair $(i,|Q|-i)$. This encoding verifies:

$$
\ulcorner q\urcorner \leq\left\ulcorner q^{\prime}\right\urcorner \quad \text { iff } \quad q=q^{\prime} .
$$

In order to implement unary and split rules from $\mathcal{B}$ in $\mathcal{B}^{\prime}$, we actually introduce six new coordinates. For a state $q$ in $Q$ and $0 \leq k \leq 2$, we write $\ulcorner q\urcorner k$ for the concatenation $0^{2 k} \cdot\ulcorner q\urcorner \cdot 0^{2(2-k)+d}$. For a vector $\overline{\mathrm{u}}$ in $\mathbb{Z}^{d}$, we also write $\overline{\mathrm{u}}$ for its concatenation with $0^{6}$ on its left.

Let us define $\mathcal{B}^{\prime}$ :

$$
\begin{aligned}
& T_{u}^{\prime} \stackrel{\text { def }}{=}\left\{\ulcorner q\urcorner^{0}-\left\ulcorner q_{1}\right\urcorner^{1}-\overline{\mathrm{u}} \mid q \stackrel{\overline{\mathrm{u}}}{\rightarrow} q_{1}\right\}, \\
& \cup\left\{\ulcorner q\urcorner^{1}-\ulcorner q\urcorner^{0} \mid q \in Q\right\} \cup\left\{\ulcorner q\urcorner^{2}-\ulcorner q\urcorner^{0} \mid q \in Q\right\}, \\
& T_{s}^{\prime} \stackrel{\text { def }}{=}\left\{\ulcorner q\urcorner^{0}-\left\ulcorner q_{1}\right\urcorner^{1}-\left\ulcorner q_{2}\right\urcorner^{2} \mid q \rightarrow q_{1}+q_{2}\right\}, \overline{\mathrm{v}}_{r} \stackrel{\text { deff }}{=}\left\ulcorner q_{r}\right\urcorner^{0}, \\
& \overline{\mathrm{v}}_{\ell} \stackrel{\text { def }}{=}\left\ulcorner q_{\ell}\right\urcorner^{0} .
\end{aligned}
$$

Observe that $\left\|T_{u}^{\prime}\right\|$ is in $O\left(|Q|+\left\|T_{u}\right\|\right)$, while $\left\|T_{s}^{\prime}\right\|,\left\|v_{r}\right\|$ and $\left\|v_{\ell}\right\|$ are in $O(|Q|)$. We prove the correction of this construction by a series of claims:

Claim B.1. If $\mathcal{B}, q_{\ell} \triangleright q, \bar{v}$, then $\mathcal{B}^{\prime}, \overline{\mathrm{v}}_{\ell} \triangleright\ulcorner q\urcorner 0+\overline{\mathrm{v}}$.

By induction on the structure of a proof for the judgement $\mathcal{B}, q_{\ell} \triangleright q, \bar{v}$.

- For the base case, i.e. $\mathcal{B}, q_{\ell} \triangleright q_{\ell}, \overline{0}$, then $\mathcal{B}^{\prime}, \bar{v}_{\ell} \triangleright \bar{v}_{\ell}=\left\ulcorner q_{\ell}\right\urcorner 0+\overline{0}$ by (16).

- For the induction step, first assume $\mathcal{B}, q_{\ell} \triangleright q, \bar{v}$ as the result of a unary rule $q \stackrel{u}{\rightarrow} q_{1}$. Then by induction hypothesis, $\mathcal{B}^{\prime}, \bar{v}_{\ell} \triangleright\left\ulcorner q_{1}\right\urcorner^{0}+\overline{\mathrm{v}}+\overline{\mathrm{u}}$. Applying (13) yields $\mathcal{B}^{\prime}, \bar{v}_{\ell} \triangleright\left\ulcorner q_{1}\right\urcorner^{1}+\bar{v}+\bar{u}$, from which (12) shows $\mathcal{B}^{\prime}, \bar{v}_{\ell} \triangleright\ulcorner q\urcorner^{0}+\bar{v}$ as desired. 
- Still for the induction step, assume the root judgement results from a split rule $q \rightarrow q_{1}+q_{2}$. Then by induction hypothesis, $\mathcal{B}^{\prime}, \overline{\mathbf{v}}_{\ell} \triangleright\left\ulcorner q_{1}\right\urcorner^{0}+$ $\overline{\mathrm{v}}_{1}$, from which (13) yields $\mathcal{B}^{\prime}, \overline{\mathrm{v}}_{\ell} \triangleright\left\ulcorner q_{1}\right\urcorner^{1}+\overline{\mathrm{v}}_{1}$, and also by induction hypothesis $\mathcal{B}^{\prime}, \overline{\mathrm{v}}_{\ell} \triangleright\left\ulcorner q_{2}\right\urcorner^{0}+\overline{\mathrm{v}}_{2}$, from which (13) yields $\mathcal{B}^{\prime}, \overline{\mathrm{v}}_{\ell} \triangleright\left\ulcorner q_{2}\right\urcorner^{2}+$ $\overline{\mathrm{v}}_{2}$. Applying (14) then shows $\mathcal{B}^{\prime}, \overline{\mathrm{v}}_{\ell} \triangleright\ulcorner q\urcorner^{0}+\overline{\mathrm{v}}_{1}+\overline{\mathrm{v}}_{2}$ as desired.

Claim B.2. If $0 \leq k \leq 2$ and $\mathcal{B}^{\prime}, \bar{v}_{\ell} \triangleright\ulcorner q\urcorner k+\bar{v}$, then $\mathcal{B}, q_{\ell} \triangleright q, \bar{v}$.

By induction on the structure of a proof for the judgement $\mathcal{B}^{\prime}, \bar{v}_{\ell} \triangleright\ulcorner q\urcorner k+\bar{v}$.

- For the base case, $\mathcal{B}^{\prime}, \bar{v}_{\ell} \triangleright\left\ulcorner q_{\ell}\right\urcorner^{0}$ by (16), and indeed $\mathcal{B}, q_{\ell} \triangleright q_{\ell}, \overline{0}$.

- For the induction step, if the judgement results from (12), then $\mathcal{B}^{\prime}, \overline{\mathrm{v}}_{\ell} \triangleright\left\ulcorner q_{1}\right\urcorner^{1}+\overline{\mathrm{v}}+\overline{\mathrm{u}}$ with $k=0$. By induction hypothesis $\mathcal{B}, q_{\ell} \triangleright$ $q_{1}, \overline{\mathrm{v}}+\overline{\mathrm{u}}$, from which $q \stackrel{\overline{\mathrm{u}}}{\rightarrow} q_{1}$ yields the desired root label.

- If the judgement results from (13), then $k>0$ and the judgement has $\mathcal{B}^{\prime}, \bar{v}_{\ell} \triangleright\ulcorner q\urcorner^{0}+\bar{v}$ as premise, and the induction hypothesis allows to conclude directly.

- If the judgement results from (14), then $\mathcal{B}^{\prime}, \overline{\mathrm{v}}_{\ell} \triangleright\left\ulcorner q_{1}\right\urcorner^{1}+\overline{\mathrm{v}}_{1}$ and $\mathcal{B}^{\prime}, \overline{\mathbf{v}}_{\ell} \triangleright\left\ulcorner q_{2}\right\urcorner^{2}+\overline{\mathrm{v}}_{2}$. By the induction hypothesis $\mathcal{B}, q_{\ell} \triangleright q_{1}, \overline{\mathrm{v}}_{1}$ and $\mathcal{B}, q_{\ell} \triangleright q_{2}, \bar{v}_{2}$, and an application of $q \rightarrow q_{1}+q_{2}$ yields the desired result.

In order to conclude on the correction, observe by an easy induction that $\mathcal{B}^{\prime}, \overline{\mathrm{v}}_{\ell} \triangleright \overline{\mathrm{w}}$ implies $\overline{\mathrm{w}}=\ulcorner q\urcorner^{k}+\overline{\mathrm{v}}$ for some $0 \leq k \leq 2, q$ in $Q$, and $\overline{\mathrm{v}}$ in $\mathbb{N}^{d}$. Thus $\overline{\mathrm{w}} \geq \overline{\mathrm{v}}_{r}$ if and only if $\overline{\mathrm{w}}=\left\ulcorner q_{r}\right\urcorner^{0}+\overline{\mathrm{v}}$ for some $\overline{\mathrm{v}}$ in $\mathbb{N}^{d}$, and by the previous claims and (11), if and only if $\mathcal{B}, q_{\ell} \triangleright q_{r}, \overline{\mathrm{v}}$.

Finally, regarding complexity, Demri et al. [10] define in the proof of their Theorem $8 L \stackrel{\text { def }}{=}\left\|T_{u}^{\prime} \cup T_{s}^{\prime}\right\|+\left\|\overline{\mathbf{v}}_{r}\right\|+2$, which is in $O\left(|Q|+\left\|T_{u}\right\|\right), H \stackrel{\text { def }}{=} L^{(3 d) !}$, and $B \stackrel{\text { def }}{=} H^{2}$, and show that, if $\mathcal{B}^{\prime}, \overline{\mathrm{v}}_{\ell} \triangleright \overline{\mathrm{w}} \geq \overline{\mathrm{v}}_{r}$, then there is a proof of the judgement of height at most $H$ and using vector values truncable to $B$. The existence of such a proof can be established with an alternating Turing machine working in space $\log B$. Since $\log B$ is in $2^{O\left(d \cdot \log d \cdot \log \log \left(|Q|+\left\|T_{u}\right\|\right)\right)}$, this yields the stated bound on the complexity.

\section{REFERENCES}

[1] A. R. Anderson and N. D. Belnap, Jr. Entailment: The Logic of Relevance and Necessity, volume I. Princeton University Press, 1975.

[2] J.-M. Andreoli. Logic programming with focusing proofs in linear logic. J. Logic Comput., 2(3):297-347, 1992. doi:10.1093/logcom/2.3.297.

[3] K. Bimbó and J. M. Dunn. On the decidability of implicational ticket entailment. J. Symb. Log., 78(1):214-236, 2013. doi:10.2178/jsl.7801150.

[4] M. Bojańczyk, A. Muscholl, T. Schwentick, and L. Segoufin. Two-variable logic on data trees and XML reasoning. J. ACM, 56(3):1-48, 2009. doi:10.1145/ 1516512.1516515 .

[5] A. Bouajjani and M. Emmi. Analysis of recursively parallel programs. ACM Trans. Prog. Lang. Syst., 35(3):10:1-10:49, 2013. doi:10.1145/2518188.

[6] A. Church. The weak theory of implication. In A. Menne, A. Wilhelmy, and H. Angsil, editors, Kontrolliertes Denken, Untersuchungen zum Logikkalkül und zur Logik der Einzelwissenschaften, pages 22-37. Kommissions-Verlag Karl Alber, Munich, 1951.

[7] H. H. Curry and R. Feys. Combinatory Logic, Vol. 1, volume 22 of Studies in Logic and the Foundations of Mathematics. North-Holland Publishing Company, 1958.

[8] P. de Groote. Towards abstract categorial grammars. In ACL 2001, pages 252-259. ACL Press, 2001. doi:10.3115/1073012.1073045 
[9] P. de Groote, B. Guillaume, and S. Salvati. Vector addition tree automata. In LICS 2004, pages 64-73. IEEE Computer Society, 2004. doi:10.1109/LICS.2004.51.

[10] S. Demri, M. Jurdziński, O. Lachish, and R. Lazić. The covering and boundedness problems for branching vector addition systems. J. Comput. Syst. Sci., 79(1):23-38, 2013. doi:10.1016/j.jcss.2012.04.002

[11] J. Dimino, F. Jacquemard, and L. Segoufin. $\mathrm{FO}^{2}(<,+1, \sim)$ on data trees, data tree automata and branching vector addition systems. Research report, INRIA, 2015. URL http://hal.inria.fr/hal-00769249

[12] J. M. Dunn and G. Restall. Relevance logic. In D. M. Gabbay and F. Guenthner, editors, Handbook of Philosophical Logic, volume 6, pages 1-128. Kluwer Academic Publishers, 2002. doi:10.1007/978-94-017-0460-1_1

[13] D. Figueira, S. Figueira, S. Schmitz, and Ph. Schnoebelen. Ackermannian and primitive-recursive bounds with Dickson's Lemma. In LICS 2011, pages 269-278. IEEE Computer Society, 2011. doi:10.1109/LICS.2011.39

[14] S. A. Kripke. The problem of entailment. In ASL 1959, volume 24 of J. Symb. Log., page 324, 1959. (Abstract).

[15] R. Lazić and S. Schmitz. Non-elementary complexities for branching VASS, MELL, and extensions. ACM Trans. Comput. Logic, 16(3:20):1-30, 2015. doi:10.1145/ 2733375

[16] P. Lincoln, J. Mitchell, A. Scedrov, and N. Shankar. Decision problems for propositional linear logic. Ann. Pure App. Logic, 56(1-3):239-311, 1992. doi:10.1016/ 0168-0072(92)90075-B

[17] R. Majumdar and Z. Wang. Expand, enlarge, and check for branching vector addition systems. In P. R. D'Argenio and H. Melgratti, editors, Concur 2013, volume 8052 of LNCS, pages 152-166. Springer, 2013. doi:10.1007/978-3-642-40184-8_12.

[18] E. W. Mayr and A. R. Meyer. The complexity of the word problems for commutative semigroups and polynomial ideals. Adv. Math., 46(3):305-329, 1982. doi:10.1016/ 0001-8708(82)90048-2

[19] S. K. Moh. The deduction theorems and two new logical systems. Methodos, 2:56-75, 1950 .

[20] V. Padovani. Ticket entailment is decidable. Math. Struct. Comput. Sci., 23(3): 568-607, 2013. doi:10.1017/S0960129512000412

[21] O. Rambow. Multiset-valued linear index grammars: imposing dominance constraints on derivations. In $A C L$ '94, pages 263-270. ACL Press, 1994. doi:10.3115/ 981732.981768

[22] J. Riche and R. K. Meyer. Kripke, Belnap, Urquhart and relevant decidability \& complexity. In G. Gottlob, É. Grandjean, and K. Seyr, editors, CSL '98, volume 1584 of LNCS, pages 224-240. Springer, 1999. doi:10.1007/10703163_16

[23] S. Salvati. Minimalist grammars in the light of logic. In S. Pogodalla, M. Quatrini, and C. Retoré, editors, Logic and Grammar, volume 6700 of LNCS, pages 81-117. Springer, 2011. doi:10.1007/978-3-642-21490-5_5

[24] S. Schmitz. On the computational complexity of dominance links in grammatical formalisms. In ACL 2010, pages 514-524. ACL Press, 2010.

[25] R. Statman. Intuitionistic propositional logic is polynomial-space complete. Theor. Comput. Sci., 9(1):67-72, 1979. doi:10.1016/0304-3975(79)90006-9.

[26] A. Urquhart. The undecidability of entailment and relevant implication. J. Symb. Log., 49(4):1059-1073, 1984. doi:10.2307/2274261.

[27] A. Urquhart. The complexity of decision procedures in relevance logic. In J. M. Dunn and A. Gupta, editors, Truth or Consequences: Essays in honour of Nuel Belnap, pages 61-76. Kluwer Academic Publishers, 1990. doi:10.1007/978-94-009-0681-5_5.

[28] A. Urquhart. The complexity of decision procedures in relevance logic II. J. Symb. Log., 64(4):1774-1802, 1999. doi:10.2307/2586811.

[29] K. N. Verma and J. Goubault-Larrecq. Karp-Miller trees for a branching extension of VASS. Discrete Math. Theor. Comput. Sci., 7(1):217-230, 2005.

ENS CACHAN \& INRIA, France

Email address: schmitz@lsv.ens-cachan.fr 